Mathematical Models and Methods in Applied Sciences

Vol. 15, No. 7 (2005) 985-1008

(C) World Scientific Publishing Company

\title{
HIERARCHICAL MODELING BASED ON MIXED PRINCIPLES: ASYMPTOTIC ERROR ESTIMATES
}

\author{
ALEXANDRE L. MADUREIRA \\ Laboratório Nacional de Computação Científica \\ Petrópolis, RJ 25651-070, Brazil \\ alm@lncc.br
}

Received 18 September 2003

Communicated by D. Arnold

\begin{abstract}
We analyze approximation properties of dimension reduction models that are based on mixed principles. The problems of interest are elliptic PDEs in thin domains. The goal is to obtain estimates that take into account both the thickness of the domain and the order of the model. The techniques involved do not require the models to be energy minimizers, and are based on asymptotic expansions for the exact and model solutions. We obtain estimates in several norms and semi-norms, and also interior estimates (which disregards boundary layers).
\end{abstract}

Keywords: Dimension reduction; hierarchical modeling; plate.

AMS Subject Classification: 35J05, 74K20, 74G10

\section{Introduction}

The idea of taking advantage of the small thickness, and using dimension reduction to approximate PDEs that are posed in thin domains is quite attractive. In fact, it seems quite natural to pose and solve a modified problem in a region with one less dimension and then extend the reduced solution to the more general domain. Estimating how good such approximations is nontrivial.

There were several attempts to derive such modeling error estimates, but they often required the models to be of minimum energy type. ${ }^{21,24,27-29}$ To the best of our knowledge, the only exceptions were estimates based on the two energies principle, ${ }^{1,9,23,25}$ which works only for a few models, and yields estimates only in the energy norm. Such restriction did not allow for a more general analysis of hierarchical models based on mixed principles.

Arnold and Madureira ${ }^{3}$ investigated minimum energy models for a Poisson problem in thin plates, and described how the models converge as the thickness decreases, and as the models become more sophisticated. Here, we perform similar analysis, but this time the models do not minimize the potential energy, and therefore they are not Ritz projections of the exact solution. We choose our models 
based on a mixed, or saddle point, principle. This is possible since, unlike previous analyses, we do not rely on the fact that the solution of the model is an energy minimizer. ${ }^{19}$

Consider the three-dimensional plate $P^{\varepsilon}=\Omega \times(-\varepsilon, \varepsilon)$, where $\Omega$ is a twodimensional smoothly bounded region and $\varepsilon<1$ is a small positive quantity. We denote the lateral boundary of the plate by $\partial P_{L}^{\varepsilon}=\partial \Omega \times(-\varepsilon, \varepsilon)$, and its top and bottom by $\partial P_{ \pm}^{\varepsilon}=\Omega \times\{-\varepsilon, \varepsilon\}$. Let $u^{\varepsilon} \in H^{1}\left(P^{\varepsilon}\right)$ be the weak solution of

$$
\begin{aligned}
-\Delta u^{\varepsilon} & =f^{\varepsilon} & & \text { in } P^{\varepsilon}, \\
\frac{\partial u^{\varepsilon}}{\partial n} & =g^{\varepsilon} & & \text { on } \partial P_{ \pm}^{\varepsilon}, \\
u^{\varepsilon} & =0 & & \text { on } \partial P_{L}^{\varepsilon},
\end{aligned}
$$

where $f^{\varepsilon}: P^{\varepsilon} \rightarrow \mathbb{R}$ and $g^{\varepsilon}: P_{ \pm}^{\varepsilon} \rightarrow \mathbb{R}$.

There is an alternative way to characterize $u^{\varepsilon}$. Let $V\left(P^{\varepsilon}\right)=L^{2}\left(P^{\varepsilon}\right)$ and $\underline{S}_{g^{\varepsilon}}\left(P^{\varepsilon}\right)=\left\{\underline{\sigma} \in \underline{H}\left(\operatorname{div}, P^{\varepsilon}\right): \underline{\sigma} \cdot \underline{n}=g^{\varepsilon}\right.$ on $\left.\partial P_{ \pm}^{\varepsilon}\right\}$. Define $\underline{\sigma}^{\varepsilon}=\left(\sigma_{\sim}^{\varepsilon}, \sigma_{3}^{\varepsilon}\right)=\underline{\nabla} u^{\varepsilon}$. Then, $\left(u^{\varepsilon}, \underline{\sigma}^{\varepsilon}\right)$ is the unique critical point of

$$
\mathcal{L}(v, \underline{\tau})=\frac{1}{2} \int_{P^{\varepsilon}}|\underline{\tau}|^{2} d \underline{x}^{\varepsilon}+\int_{P^{\varepsilon}} f^{\varepsilon} v d \underline{x}^{\varepsilon}+\int_{P^{\varepsilon}} \operatorname{div} \underline{\tau} v d \underline{x}^{\varepsilon}
$$

on $V\left(P^{\varepsilon}\right) \times S_{g^{\varepsilon}}\left(P^{\varepsilon}\right)$. Actually, $\left(u^{\varepsilon}, \sigma^{\varepsilon}\right)$ is a saddle point of $\mathcal{L}$.

We introduce now two classes of models based on the above variational principle. Choosing subspaces of $V\left(P^{\varepsilon}\right)$ and $\underline{S}_{g^{\varepsilon}}\left(P^{\varepsilon}\right)$ composed of functions with polynomial dependence in the transverse direction, and looking for a critical point of $\mathcal{L}$ within these subspaces, we define saddle point models which we call $\mathrm{SP}^{\prime}$ models. For a function $v \in L^{2}\left(P^{\varepsilon}\right)$, and an integer $p$, we write $\operatorname{deg}_{3} v \leq p$ meaning that $v$ is a polynomial of degree at most $p$ in $x_{3}$, with coefficients in $L^{2}(\Omega)$. The interpretation for $\operatorname{deg}_{3} v<0$ is that $v=0$. For $V\left(P^{\varepsilon}, p\right)=\left\{v \in V\left(P^{\varepsilon}\right): \operatorname{deg}_{3} v \leq p\right\}$ and

$$
\underline{S}_{g^{\varepsilon}}\left(P^{\varepsilon}, p\right)=\left\{\underline{\tau} \in \underline{S}_{g^{\varepsilon}}\left(P^{\varepsilon}\right): \operatorname{deg}_{3} \underset{\sim}{\sim} \leq p, \operatorname{deg}_{3} \tau_{3} \leq p-1\right\}
$$

we have the $S P_{1}^{\prime}(p)$ models. Another option is to choose $\underline{S}_{g^{\varepsilon}}\left(P^{\varepsilon}, p\right)=\{\underline{\tau} \in$ $\left.\underline{S}_{g}\left(P^{\varepsilon}\right): \operatorname{deg}_{3} \tau \leq p, \operatorname{deg}_{3} \tau_{3} \leq p+1\right\}$, and we define the $\bar{S} P_{2}^{\prime}(p)$ models. The solutions of the models, $u^{\varepsilon}(p) \in V\left(P^{\varepsilon}, p\right)$ and $\underline{\sigma}^{\varepsilon}(p) \in \underline{S}_{g^{\varepsilon}}\left(P^{\varepsilon}, p\right)$ satisfy the weak equations

$$
\begin{array}{ll}
\int_{P^{\varepsilon}} \underline{\sigma}^{\varepsilon}(p) \cdot \underline{\tau} d \underline{x}^{\varepsilon}+\int_{P^{\varepsilon}} u^{\varepsilon}(p) \operatorname{div} \underline{\tau} d \underline{x}^{\varepsilon}=0 & \text { for all } \underline{\tau} \in \underline{S}_{0}\left(P^{\varepsilon}, p\right), \\
\int_{P^{\varepsilon}} \operatorname{div} \underline{\sigma}^{\varepsilon}(p) v d \underline{x}^{\varepsilon}=-\int_{P^{\varepsilon}} f^{\varepsilon} v d \underline{x}^{\varepsilon} & \text { for all } v \in V\left(P^{\varepsilon}, p\right) .
\end{array}
$$

Note that in both $\mathrm{SP}_{1}^{\prime}(p)$ and $\mathrm{SP}_{2}^{\prime}(p)$ models, $\operatorname{div} S_{g^{\varepsilon}}\left(P^{\varepsilon}, p\right)=V\left(P^{\varepsilon}, p\right)$ and therefore, not only there exists a unique solution for (1.2), (1.3), but also $\operatorname{div} \sigma^{\varepsilon}(p)=$ $-\pi_{V} f^{\varepsilon}$, where $\pi_{V} f^{\varepsilon}$ is the orthogonal $L^{2}$ projection of $f^{\varepsilon}$ into $V\left(P^{\varepsilon}, p\right)$. This implies that $\sigma^{\varepsilon}(p)$ is the minimizer of the complementary energy

$$
\mathcal{J}_{c}(\underline{\tau})=\frac{1}{2} \int_{P^{\varepsilon}}|\underline{\tau}|^{2} d \underline{x}
$$

over all $\underline{\tau} \in \underline{S}_{g^{\varepsilon}}\left(P^{\varepsilon}, p\right)$ such that $\operatorname{div} \underline{\tau}=-\pi_{V} f^{\varepsilon}$. 
It is clear that by increasing $p$, we generate two families of hierarchical models, $\mathrm{SP}_{1}^{\prime}(p)$ and $\mathrm{SP}_{2}^{\prime}(p)$. We study here the more sophisticated $\mathrm{SP}_{2}^{\prime}(p)$ models. The simplest instance in this family, $\mathrm{SP}_{2}^{\prime}(1)$, follows. If

$$
\begin{aligned}
& u^{\varepsilon}(1)\left(\underline{x}^{\varepsilon}\right)=\omega_{0}(\underset{\sim}{x})+\omega_{1}\left({\underset{\sim}{x}}^{\varepsilon}\right) x_{3}^{\varepsilon}, \\
& \underline{\sigma}^{\varepsilon}(1)\left(\underline{x}^{\varepsilon}\right)=\left(\begin{array}{c}
\underset{\sim}{\nabla} \omega_{0}\left({\underset{\sim}{x}}^{\varepsilon}\right) \\
\varepsilon^{-1} g^{0} x_{3}^{\varepsilon}
\end{array}\right)+\left(\begin{array}{c}
\underset{\sim}{\nabla} \omega_{1}\left({\underset{\sim}{x}}^{\varepsilon}\right) x_{3}^{\varepsilon} \\
-\frac{5}{4}\left(\varepsilon^{-2} x_{3}^{\varepsilon 2}-1\right) \omega_{1}+\frac{1}{4}\left(5 \varepsilon^{-2} x_{3}^{\varepsilon}-1\right) g^{1}
\end{array}\right),
\end{aligned}
$$

then

$$
\begin{aligned}
& \Delta_{2 D} \omega_{0}=-f^{0}-\varepsilon^{-1} g^{0}, \quad \frac{2}{3} \varepsilon^{2} \Delta_{2 D} \omega_{1}-\frac{5}{3} \omega_{1}=-2 f^{1}-\frac{5}{3} g^{1} \quad \text { in } \Omega, \\
& \omega_{0}=\omega_{1}=0 \quad \text { on } \partial \Omega,
\end{aligned}
$$

where $\Delta_{2 D}=\partial_{11}+\partial_{22}$ and

$$
\begin{aligned}
& f^{0}\left({\underset{\sim}{x}}^{\varepsilon}\right)=\frac{1}{2 \varepsilon} \int_{-\varepsilon}^{\varepsilon} f^{\varepsilon}\left({\underset{\sim}{x}}^{\varepsilon}, x_{3}^{\varepsilon}\right) d x_{3}^{\varepsilon}, \quad f^{1}\left({\underset{\sim}{\varepsilon}}^{\varepsilon}\right)=\frac{1}{2 \varepsilon} \int_{-\varepsilon}^{\varepsilon} f^{\varepsilon}\left({\underset{\sim}{x}}^{\varepsilon}, x_{3}^{\varepsilon}\right) x_{3}^{\varepsilon} d x_{3}^{\varepsilon}, \\
& g^{0}\left(\underset{\sim}{x^{\varepsilon}}\right)=\frac{1}{2}\left[g^{\varepsilon}\left({\underset{\sim}{x}}^{\varepsilon}, \varepsilon\right)+g^{\varepsilon}(\underset{\sim}{x},-\varepsilon)\right], \quad g^{1}(\underset{\sim}{x})=\frac{1}{2}\left[g^{\varepsilon}(\underset{\sim}{x}, \varepsilon)-g^{\varepsilon}\left({\underset{\sim}{x}}^{\varepsilon},-\varepsilon\right)\right] .
\end{aligned}
$$

The two differential equations in (1.4) are independent of each other. We can express any function defined on $P^{\varepsilon}$ as a sum of its even and odd parts with respect to $x_{3}^{\varepsilon}$, in a unique way. The even parts of $f^{\varepsilon}, g^{\varepsilon}$ appear only in the equation for $\omega_{0}$, and the respective odd parts show up in the equation for $\omega_{1}$. Also, the equation determining $\omega_{1}$ is singularly perturbed, but this is not the case for the equation determining $\omega_{0}$. If higher order methods were used, we would have two independent singularly perturbed systems of equations, corresponding to the even and odd parts of $u^{\varepsilon}(p)$. A similar splitting also occurs for the linearly elastic isotropic and homogeneous plate, where the equations decouple into two independent problems corresponding to bending and stretching of the plate.

We denote by $\mathbb{P}_{p}(-a, a)$ the space of polynomials of degree $p$ in $(-a, a)$. Also, if $s$ is a real number and $D$ is an open domain, then $H^{s}(D)$ is the Sobolev space of order $s$, and $\stackrel{\circ}{H}^{s}(D)$ is the closure in $H^{s}(D)$ of the set of smooth functions with compact support. We write $\hat{L}^{2}(-a, a)$ to indicate the set of square integrable functions with mean value zero in the domain $(-a, a)$, for a positive number $a$. For $m \in \mathbb{N}$ and a certain separable Hilbert space $E, H^{m}(D ; E)$ is the space of functions defined on $D$ with values in $E$ such that the $E$-norm of all partial derivatives of order less than or equal to $m$ are in $L^{2}(D)$. And $\mathcal{D}(D)$ denotes the space of $\mathcal{C}^{\infty}$ functions in $D$ with compact support, while $\mathcal{D}^{\prime}(D)$ is the space of distributions.

As we have already hinted, we use one underbar for 3-vectors and one undertilde for 2 -vectors. We can then decompose 3 -vectors as follows:

$$
\underline{u}=\left(\begin{array}{c}
u \\
\sim \\
u_{3}
\end{array}\right) .
$$

We denote a typical point in $P^{\varepsilon}$ by $\underline{x}^{\varepsilon}=\left({\underset{\sim}{x}}^{\varepsilon}, x_{3}^{\varepsilon}\right)$, with ${\underset{\sim}{x}}^{\varepsilon}=\left(x_{1}^{\varepsilon}, x_{2}^{\varepsilon}\right) \in \Omega$. 
The goal of this work is to estimate the modeling error, and we do so by comparing the asymptotic expansions of $u^{\varepsilon}$ and $u^{\varepsilon}(p)$. The expansion for $u^{\varepsilon}$ was carefully developed in the work of Arnold and Madureira, ${ }^{3}$ and we summarize the main results in Sec. 2. In this same section, we develop the expansion for $u^{\varepsilon}(p)$, and present related estimates. In Sec. 3 we obtain the modeling errors in several norms. Finally, in the Appendix, we deal with issues related to approximation properties at the boundary layers present in the solution of (1.1).

\section{Asymptotic Expansions}

To introduce the asymptotic expansion for $u^{\varepsilon}$, some notation is necessary. For each point $x^{\varepsilon}$ in $P^{\varepsilon}$ we assign a point $x=\left(\underset{x}{x}, x_{3}\right)=\left({\underset{x}{\varepsilon}}^{\varepsilon}, \varepsilon^{-1} x_{3}^{\varepsilon}\right)$ in the $\varepsilon$-independent plate $\bar{P}=\Omega \times(-1,1)$. We also define $\left.\partial \widetilde{P_{L}}=\partial \Omega \times \widetilde{(}-1,1\right)$ and $\partial P_{ \pm}=\Omega \times\{-1,1\}$. In this new domain, we define $f(\underline{x})=f^{\varepsilon}\left(\underline{x}^{\varepsilon}\right)$ and $g(\underline{x})=\varepsilon^{-1} g^{\varepsilon}\left(\underline{x}^{\varepsilon}\right)$.

It is possible to formally expand $u^{\varepsilon}$ in the power series

$$
u^{\varepsilon}\left(\underline{x}^{\varepsilon}\right) \sim \sum_{k=0}^{\infty} \varepsilon^{2 k} u^{2 k}\left({\underset{\sim}{x}}^{\varepsilon}, \varepsilon^{-1} x_{3}^{\varepsilon}\right)-\chi(\rho) \sum_{k=2}^{\infty} \varepsilon^{k} \tilde{U}^{k}\left(\varepsilon^{-1} \rho, \theta, \varepsilon^{-1} x_{3}^{\varepsilon}\right) .
$$

The leading term $u^{0}\left(\underline{x}^{\varepsilon}\right)=\zeta^{0}\left({\underset{\sim}{x}}^{\varepsilon}\right)$, where

$$
\begin{aligned}
\Delta_{2 D} \zeta^{0}(\underset{\sim}{x}) & =-\frac{1}{2} \int_{-1}^{1} f\left(\underset{\sim}{x}, x_{3}\right) d x_{3}-\frac{1}{2}[g(\underset{\sim}{x}, 1)+g(\underset{\sim}{x},-1)], \\
\zeta^{0} & =0 \quad \text { on } \partial \Omega .
\end{aligned}
$$

For all $\underset{\sim}{x} \in \Omega$,

$$
\begin{aligned}
\partial_{33} u^{2} & =-f-\Delta_{2 D} u^{0}, \quad \text { in }(-1,1), \\
\int_{-1}^{1} u^{2}\left(\underset{\sim}{x}, x_{3}\right) d x_{3} & =0, \quad \partial_{3} u^{2}(\underset{\sim}{x},-1)=-g(\underset{\sim}{x},-1), \quad \partial_{3} u^{2}(\underset{\sim}{x}, 1)=g(\underset{\sim}{x}, 1) .
\end{aligned}
$$

Similarly, for $k>1$,

$$
\begin{aligned}
\partial_{33} u^{2 k} & =-\Delta_{2 D} u^{2 k-2}, \quad \text { in }(-1,1), \\
\int_{-1}^{1} u^{2 k}\left(\underset{\sim}{x}, x_{3}\right) d x_{3} & \left.=0, \quad \partial_{3} u^{2 k}(\underset{\sim}{x},-1)=\partial_{3} u^{2 k} \underset{\sim}{x}, 1\right)=0 .
\end{aligned}
$$

The functions $\tilde{U}^{k}$ are boundary correctors, functions that decay exponentially fast away from the lateral boundary, indicating the presence of boundary layers in the original solution. These functions are defined only close to the lateral boundary $\partial P_{L}^{\varepsilon}$, and can be expressed in a simpler form if we use a local coordinate system. So, we indicate a point $\underset{\sim}{x^{\varepsilon}}$ close enough to $\partial \Omega$ by $(\rho, \theta)$, where $\rho<\rho_{0}$ is the distance between $\underset{\sim}{x}$ and $\partial \Omega, \rho_{0}$ is a positive number smaller than the minimum radius of curvature, and $\theta$ gives roughly the arclength along the boundary. ${ }^{2,3,9}$ The boundary 
correctors $\tilde{U}^{k}$ are originally defined in the semi-infinite strip $\Sigma=\mathbb{R}^{+} \times(-1,1)$, as the solutions of

$$
\begin{aligned}
\left(\partial_{\tilde{\rho} \tilde{\rho}}+\partial_{33}\right) \tilde{U}^{k} & =F_{k} & & \text { in } \Sigma \\
\frac{\partial \tilde{U}^{k}}{\partial n} & =0 & & \text { on } \mathbb{R}^{+} \times\{-1,1\}, \\
\tilde{U}^{k}\left(0, \theta, x_{3}\right) & =u^{k}\left(0, \theta, x_{3}\right) & & \text { for } x_{3} \in(-1,1),
\end{aligned}
$$

where

$$
\begin{aligned}
& a_{1}^{j}=-[\kappa(\theta)]^{j+1}, \quad a_{2}^{j}=(j+1)[\kappa(\theta)]^{j}, \quad a_{3}^{j}=\frac{j(j+1)}{2}[\kappa(\theta)]^{j-1} \kappa^{\prime}(\theta), \\
& F_{k}=\sum_{j=0}^{k-2} \tilde{\rho}^{j}\left(a_{1}^{j} \partial_{\tilde{\rho}} \tilde{U}^{k-j-1}+a_{2}^{j} \partial_{\theta \theta} \tilde{U}^{k-j-2}+a_{3}^{j} \partial_{\theta} \tilde{U}^{k-j-2}\right),
\end{aligned}
$$

with the convention that $u^{k}=0$ for $k$ odd and $\tilde{U}^{0}=\tilde{U}^{1}=0$.

Finally, $\chi(\rho)$ is a smooth cutoff function identically one for $0 \leq \rho \leq \rho_{0} / 3$ and zero for $\rho \geq 2 \rho_{0} / 3$. The introduction of $\chi$ allows the definition of the boundary correctors on all $P^{\varepsilon}$, and only adds an error that decays exponentially with $\varepsilon^{-1}$.

We begin to compile several results that are useful. The proofs can be found elsewhere. ${ }^{3,19}$ First we bound Sobolev norms for some terms in the asymptotic expansion. These bounds follow immediately from regularity results for Eqs. (2.1) (2.3). We denote by lowercase $c$ a generic constant (not necessarily the same in all occurrences) which is independent not only of $\varepsilon$ and $p$, but also of $f$ and $g$, while we use uppercase $C$ when the constant may depend on $f$ and $g$, more precisely on Sobolev norms of $f$ and $g$, but not $\varepsilon$ and $p$. Also,

$$
\|v\|_{(m, n, P)}=\|v\|_{H^{m}\left(\Omega ; H^{n}(-1,1)\right)}, \quad\|(f, g)\|_{m, P}=\|f\|_{(m, 0, P)}+\|g\|_{H^{m}\left(\partial P_{ \pm}\right)} .
$$

Lemma 2.1. Suppose that $f$ and $g$ are smooth functions on $P$ and $\partial P_{ \pm}$, respectively. Then the functions $u^{0}, u^{2}, \ldots$ on $P$ are uniquely determined by $(2.1)-(2.3)$, and $u^{0}(\underline{x})=\zeta^{0}(\underset{\sim}{x})$ is independent of $x_{3}$. Moreover, for $m$ a non-negative integer and $s$ a real number such that $s \geq 2$, there exists a constant $c$ independent of $f$ and $g$ such that

$$
\begin{aligned}
\left\|\zeta^{0}\right\|_{H^{m+1}(\Omega)} & \leq c\|\mid(f, g)\|_{m-1, P}, \\
\left\|u^{2}(\underset{\sim}{x}, \cdot)\right\|_{H^{s}(-1,1)} & \leq c\left(\|f(\underset{\sim}{x}, \cdot)\|_{H^{s-2}(-1,1)}+|g(\underset{\sim}{x},-1)|+|g(\underset{\sim}{x}, 1)|\right), \\
\left\|u^{2}\right\|_{(m, s, P)} & \leq c\left(\|f\|_{(m, s-2, P)}+\|g\|_{H^{m}\left(\partial P_{ \pm}\right)}\right) .
\end{aligned}
$$

Next we estimate the $H^{1}\left(P^{\varepsilon}\right)$ norm of the difference between the truncated asymptotic expansions and the exact solution.

Theorem 2.1. For any positive integer $N$, there exists a constant $C$ such that the difference between the truncated asymptotic expansion and the original solution measured in the original domain is bounded as follows:

$$
\left\|e_{0}\right\|_{H^{1}\left(P^{\varepsilon}\right)} \leq C \varepsilon^{3 / 2}, \quad\left\|e_{2 N}\right\|_{H^{1}\left(P^{\varepsilon}\right)} \leq C \varepsilon^{2 N+1},
$$


where

$$
e_{2 N}^{\varepsilon}\left(\underline{x}^{\varepsilon}\right)=u^{\varepsilon}\left(\underline{x}^{\varepsilon}\right)-\sum_{k=0}^{N} \varepsilon^{2 k} u^{2 k}\left({\underset{\sim}{x}}^{\varepsilon}, \varepsilon^{-1} x_{3}^{\varepsilon}\right)+\chi(\rho) \sum_{k=2}^{2 N} \varepsilon^{k} \tilde{U}^{k}\left(\varepsilon^{-1} \rho, \theta, \varepsilon^{-1} x_{3}^{\varepsilon}\right) .
$$

We start now to develop the asymptotic expansion for the model solution. For the $\mathrm{SP}_{2}^{\prime}(p)$ methods, the relation $\sigma^{\varepsilon}(p)=\underline{\nabla} u^{\varepsilon}(p)$ does not hold in general and so we will develop asymptotic expansions for $u^{\bar{\varepsilon}}(p)$ and $\sigma^{\varepsilon}(p)$ simultaneously. We start by rewriting Eqs. (1.2) and (1.3) in the scaled domain $P$. Define

$$
\begin{aligned}
u(p)(\underline{x}) & =u^{\varepsilon}(p)\left(\underline{x}^{\varepsilon}\right), \quad \underset{\sim}{\sigma}(p)(\underline{x})={\underset{\sim}{\sigma}}^{\varepsilon}(p)\left(\underline{x}^{\varepsilon}\right), \quad \sigma_{3}(p)(\underline{x})=\varepsilon \sigma_{3}^{\varepsilon}(p)\left(\underline{x}^{\varepsilon}\right), \\
V(P, p) & =\left\{v \in L^{2}(P): \operatorname{deg}_{3} v \leq p\right\}, \\
\underline{S}_{g}(P, p) & =\left\{\underline{\tau} \in \underline{\mathcal{D}}^{\prime}(P): \underline{\tau} \in \underline{L}^{2}(P), \operatorname{div} \underset{\sim}{\tau}+\varepsilon^{-2} \partial_{3} \tau_{3} \in L^{2}(P),\right. \\
\underline{\tau} \cdot \underline{n} & \left.=g \text { on } \partial P_{ \pm}, \operatorname{deg}_{3} \underset{\sim}{\tau} \leq p, \operatorname{deg}_{3} \tau_{3} \leq p+1\right\} .
\end{aligned}
$$

Then $u(p) \in V(P, p)$ and $\underline{\sigma}(p) \in \underline{S}_{\varepsilon^{2} g}(P, p)$ satisfy

$$
\begin{aligned}
& \int_{P} \underset{\sim}{\sigma}(p) \cdot \underset{\sim}{\tau}+\varepsilon^{-2} \sigma_{3}(p) \tau_{3} d \underline{x}+\int_{P} u(p)\left(\operatorname{div} \underset{\sim}{\tau}+\varepsilon^{-2} \partial_{3} \tau_{3}\right) d \underline{x}=0 \\
& \text { for all } \tau \in \underline{S}_{0}(P, p) \text {, } \\
& \int_{P}\left[\operatorname{div} \underset{\sim}{\sigma}(p)+\varepsilon^{-2} \partial_{3} \sigma_{3}(p)\right] v d \underline{x}=-\int_{P} f v d \underline{x} \quad \text { for all } v \in V(P, p) .
\end{aligned}
$$

Consider the asymptotic expansions

$$
\begin{aligned}
& u^{0}(p)+\varepsilon^{2} u^{2}(p)+\varepsilon^{4} u^{4}(p)+\cdots, \\
& \underline{\sigma}^{0}(p)+\varepsilon^{2} \underline{\sigma}^{2}(p)+\varepsilon^{4} \underline{\sigma}^{4}(p)+\cdots,
\end{aligned}
$$

where $u^{2 k}(p) \in L^{2}(P)$ for any positive integer $k$. Also $\sigma^{0}(p)$ and $\sigma^{2 k}(p) \in S_{0}(P, p)$ for integers $k \geq 2$, and $\underline{\sigma}^{2}(p) \in \underline{S}_{g}(P, p)$. Then, after the formal process of substituting (2.8) into (2.7) and grouping together terms with the same powers of $\varepsilon$, for $k \in \mathbb{N}$ we ask that

$$
\begin{aligned}
& \int_{P} \underset{\sim}{\sigma^{2 k-2}}(p) \cdot \underset{\sim}{\tau}+\sigma_{3}^{2 k}(p) \tau_{3} d \underline{x}+\int_{P}\left[u^{2 k-2}(p) \operatorname{div} \underset{\sim}{\tau}+u^{2 k}(p) \partial_{3} \tau_{3}\right] d \underline{x}=0 \\
& \text { for all } \tau \in \underline{S}_{0}(P, p) \text {, }
\end{aligned}
$$

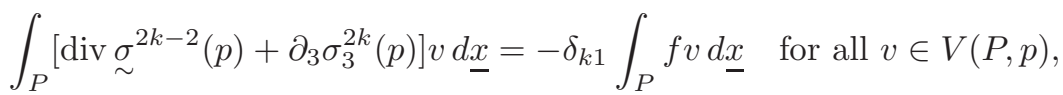

where we define $u^{-2}=0, \sigma^{-2}=0$.

We now determine $u^{2 \bar{k}}(p), \sigma^{2 k}(p)$. First, set $k=0$. From Eq. (2.10) we find that $\partial_{3} \sigma_{3}^{0}(p)=0$. As $\sigma^{0}(p) \in \bar{S}_{0}(P, p)$, then $\sigma_{3}^{0}(p)=0$. From $(2.9)$, we see that $u^{0}(p)$ is independent of $x_{3}$. Making $k=1$ and using (2.9) with $\tau_{3}=0$, it follows that $\sigma^{0}(p)=\nabla u^{0}(p)$. From the compatibility condition of $(2.10), u^{0}(p)(x)=\zeta^{0}(x)$, see $(\tilde{2.1})$. If we proceed with the argument, and with $\underset{\sim}{\sim} \in \Omega$ as a parameter, we find that $u^{2}(p)(\underset{\sim}{x}, \cdot) \in \hat{\mathbb{P}}_{p}(-1,1)$, the space of polynomials of degree $p$ in $(-1,1)$ with 
zero average. Also, $\sigma_{3}^{2}(p)(\underset{\sim}{x}, \cdot) \in \mathbb{P}_{p+1}(-1,1)$ with $\sigma_{3}^{2}(p)(\underset{\sim}{x},-1)=-g(\underset{\sim}{x},-1)$ and $\sigma_{3}^{2}(p)(\underset{\sim}{x}, 1)=g(\underset{\sim}{x}, 1)$ should satisfy

$$
\begin{aligned}
& \left.\left.\int_{-1}^{1} \sigma_{3}^{2}(p) \underset{\sim}{x}, x_{3}\right) \tau_{3}\left(x_{3}\right) d x_{3}+\int_{-1}^{1} u^{2}(p) \underset{\sim}{x}, x_{3}\right) \partial_{3} \tau_{3}\left(x_{3}\right) d x_{3}=0 \\
& \text { for all } \tau_{3} \in \stackrel{\circ}{\mathbb{P}}_{p+1}(-1,1) \text {, } \\
& \int_{-1}^{1} \partial_{3} \sigma_{3}^{2}(p)\left(\underset{\sim}{x}, x_{3}\right) v\left(x_{3}\right) d x_{3}=-\int_{-1}^{1}\left[f\left(\underset{\sim}{x}, x_{3}\right)+\Delta_{2 D} \zeta^{0}(\underset{\sim}{x})\right] v\left(x_{3}\right) d x_{3} \\
& \text { for all } v \in \hat{\mathbb{P}}_{p}(-1,1) \text {, } \\
& \stackrel{\sigma}{\sim}^{2}(p)=\underset{\sim}{\nabla} u^{2}(p)
\end{aligned}
$$

where $\stackrel{\circ}{\mathbb{P}}_{p+1}(-1,1)=\mathbb{P}_{p+1}(-1,1) \cap \stackrel{\circ}{H}^{1}(-1,1)$. Also, for any integer $k \geq 2$, we define $\sigma_{3}^{2 k}(p)(\underset{\sim}{x}, \cdot) \in \stackrel{\circ}{\mathbb{P}}_{p+1}(-1,1)$ and $u^{2 k}(p)(\underset{\sim}{x}, \cdot) \in \hat{\mathbb{P}}_{p}(-1,1)$ by imposing

$$
\begin{gathered}
\left.\int_{-1}^{1} \sigma_{3}^{2 k}(p) \underset{\sim}{x}, x_{3}\right) \tau_{3}\left(x_{3}\right) d x_{3}+\int_{-1}^{1} u^{2 k}(p)\left({\left.\underset{\sim}{x}, x_{3}\right) \partial_{3} \tau_{3}\left(x_{3}\right) d x_{3}=0}^{\text {for all } \tau_{3} \in \mathbb{P}_{p+1}(-1,1),}\right. \\
\left.\int_{-1}^{1} \partial_{3} \sigma_{3}^{2 k}(p) \underset{\sim}{x}, x_{3}\right) v\left(x_{3}\right) d x_{3}=-\int_{-1}^{1} \Delta_{2 D} u^{2 k-2}(p)\left(\underset{\sim}{x}, x_{3}\right) v\left(x_{3}\right) d x_{3} \\
\quad \text { for all } v \in \hat{\mathbb{P}}_{p+1}(-1,1), \\
\stackrel{\sigma}{\sim}^{2 k}(p)=\underset{\sim}{\nabla} u^{2 k}(p) .
\end{gathered}
$$

Note from $(2.2)$ that $u^{2}(p)(\underset{\sim}{x}, \cdot)$, and $\sigma_{3}^{2}(p)(\underset{\sim}{x}, \cdot)$ are mixed approximations for $u^{2}(\underset{\sim}{x}, \cdot)$, and $\partial_{3} u^{2}(\underset{\sim}{x}, \cdot)$, with $\underset{\sim}{x} \in \Omega$ as a parameter.

Equation (2.9) imposes, as a natural condition, that $u(p)$ vanishes on $\partial P_{L}$. Nevertheless, this boundary condition is not being imposed for the terms of the asymptotic expansion, with the exception of $u^{0}(p)$. Thus, in general, $u^{2 k}(p)$ does not vanish on $\partial P_{L}$, for $k \geq 1$. We seek then a pair of correctors $U(p)$, $\Xi(p)$ such that

$$
\begin{gathered}
\int_{P} \underset{\sim}{\Xi}(p) \cdot \underset{\sim}{\tau}+\varepsilon^{-2} \Xi_{3}(p) \tau_{3}+U(p)\left(\operatorname{div} \underset{\sim}{\tau}+\varepsilon^{-2} \partial_{3} \tau_{3}\right) d \underline{x}=\int_{\partial P_{L}} U_{0} \underset{\sim}{\tau} \cdot \underset{\sim}{n} d \underset{\sim}{d x} d x_{3} \\
\quad \text { for all } \underline{\tau} \in \underline{S}_{0}(P, p), \\
\int_{P}\left[\operatorname{div} \underset{\sim}{\Xi}(p)+\varepsilon^{-2} \partial_{3} \Xi_{3}(p)\right] v d \underline{x}=0 \quad \text { for all } v \in V(P, p), \\
U_{0} \sim \varepsilon^{2} u^{2}(p)+\varepsilon^{4} u^{4}(p)+\cdots .
\end{gathered}
$$

We rewrite the singular perturbation problem (2.13) using the horizontal boundaryfitted coordinates $(\rho, \theta)$ in the region

$$
\Omega_{b}=\left\{\underset{\sim}{z}-\rho \underset{\sim}{n}: \underset{\sim}{z} \in \partial \Omega, 0<\rho<\rho_{0}\right\},
$$


where $\rho_{0}$ is a positive number smaller than the minimum radius of curvature of $\partial \Omega$. The normal and tangential vectors to $\partial \Omega$ extend naturally to $\Omega_{b}$ by

$$
\underset{\sim}{n}(\rho, \theta)=\underset{\sim}{n}(\theta), \quad \underset{\sim}{s}(\rho, \theta)=\underset{\sim}{s}(\theta),
$$

and the quantities

$$
\begin{array}{ll}
\Xi_{n}(p)\left(\underline{x}^{\varepsilon}\right)=\underset{\sim}{\Xi}(p)\left(\underline{x}^{\varepsilon}\right) \cdot \underset{\sim}{n}\left(\underset{\sim}{x^{\varepsilon}}\right), & \Xi_{s}(p)\left(\underline{x}^{\varepsilon}\right)=\underset{\sim}{\Xi}(p)\left(\underline{x}^{\varepsilon}\right) \cdot \underset{\sim}{s}\left(\underset{\sim}{x^{\varepsilon}}\right), \\
\tau_{n}(p)\left(\underline{x}^{\varepsilon}\right)=\underset{\sim}{\tau}(p)\left(\underline{x}^{\varepsilon}\right) \cdot \underset{\sim}{n}\left({\underset{\sim}{x}}^{\varepsilon}\right), & \tau_{s}(p)\left(\underline{x}^{\varepsilon}\right)=\underset{\sim}{\tau}(p)\left(\underline{x}^{\varepsilon}\right) \cdot \underset{\sim}{s}\left({\underset{\sim}{x}}^{\varepsilon}\right),
\end{array}
$$

are then well defined in $\Omega_{b}$. A long but straightforward computation shows that

$$
\operatorname{div} \underset{\sim}{\Xi}(p)=\partial_{\rho} \Xi_{n}(p)+\frac{\partial_{\theta} \Xi_{s}(p)}{J}-\frac{\kappa}{J} \Xi_{n}(p),
$$

where $J(\rho, \theta)=1-\rho \kappa(\theta)$, and $\kappa$ is the curvature of $\partial \Omega$. Next, we use the "stretched" (in the normal and vertical directions) variables $\left(\tilde{\rho}, \theta, x_{3}\right)$, where $\tilde{\rho}=\varepsilon^{-1} \rho$, to pose an $\varepsilon$-independent sequence of corrector problems, and define

$$
\begin{aligned}
& \tilde{U}(p)\left(\tilde{\rho}, \theta, x_{3}\right)=U(p)\left(\rho, \theta, x_{3}\right), \quad \tilde{\Xi}_{n}(p)\left(\tilde{\rho}, \theta, x_{3}\right)=\varepsilon \Xi_{n}(p)\left(\rho, \theta, x_{3}^{\varepsilon}\right), \\
& \tilde{\Xi}_{s}(p)\left(\tilde{\rho}, \theta, x_{3}\right)=\Xi_{s}(p)\left(\rho, \theta, x_{3}^{\varepsilon}\right), \quad \tilde{\Xi}_{3}(p)\left(\tilde{\rho}, \theta, x_{3}\right)=\varepsilon \Xi_{3}(p)\left(\rho, \theta, x_{3}^{\varepsilon}\right) \text {. }
\end{aligned}
$$

Similar definitions hold for $\tilde{\tau}_{n}, \tilde{\tau}_{s}$ and $\tilde{\tau}_{3}$. The motivation for multiplying $\Xi_{n}(p)$ and $\Xi_{3}(p)$ by $\varepsilon$ is that we expect them to "behave" as $\varepsilon^{-1}$, after all they approximate $\partial_{\rho} \tilde{U}$ and $\partial_{3} \tilde{U}$ in $P^{\varepsilon}$. The combination of the above described transformations leads to

$$
\begin{aligned}
& \int_{\tilde{Q}}\left[\varepsilon^{-2} \tilde{\Xi}_{n}(p) \tilde{\tau}_{n}+\tilde{\Xi}_{s}(p) \tilde{\tau}_{s}+\varepsilon^{-2} \tilde{\Xi}_{3}(p) \tilde{\tau}_{3}+\tilde{U}(p)\left(\varepsilon^{-2} \partial_{\tilde{\rho}} \tilde{\tau}_{n}+\frac{\partial_{\theta} \tilde{\tau}_{s}}{J}+\varepsilon^{-2} \partial_{3} \tilde{\tau}_{3}\right)\right] J \\
& -\varepsilon^{-1} \kappa \tilde{U}(p) \tilde{\tau}_{n} d \tilde{Q}=\int_{0}^{2 \pi} \int_{-1}^{1} U_{0}(p)\left(0, \theta, x_{3}\right) \tilde{\tau}_{n}\left(0, \theta, x_{3}\right) d x_{3} d \theta, \\
& \int_{\tilde{Q}}\left[\varepsilon^{-2} \partial_{\tilde{\rho}} \tilde{\Xi}_{n}(p)+\frac{\partial_{\theta} \tilde{\Xi}_{s}(p)}{J}+\varepsilon^{-2} \partial_{3} \tilde{\Xi}_{3}-\varepsilon^{-1} \frac{\kappa}{J} \tilde{\Xi}_{n}(p)\right] v J d \tilde{Q}=0,
\end{aligned}
$$

where $\tilde{Q}=\mathbb{R}^{+} \times(0,2 \pi) \times(-1,1)$ is a semi-infinite quadrilateral domain with the union of its top and bottom boundaries given by $\partial \tilde{Q}_{ \pm}=\mathbb{R}^{+} \times(0,2 \pi) \times\{-1,1\}$, and

$$
\begin{aligned}
& \tilde{\tau} \in\left\{\underline{\tau} \in \underline{H}(\operatorname{div}, \tilde{Q}): \tau_{3}=0 \text { on } \partial \tilde{Q}_{ \pm}, \operatorname{deg}_{3} \tau \leq p, \operatorname{deg}_{3} \tau_{3} \leq p+1\right\}, \\
& v \in\left\{L^{2}(\tilde{Q}): \operatorname{deg}_{3} v \leq p\right\} .
\end{aligned}
$$

Replacing $\tilde{\tau}_{n}$ by $\tilde{\tau}_{n} / J, \tilde{\tau}_{3}$ by $\tilde{\tau}_{3} / J$ and $v$ by $v / J$, using the Taylor series of $1 / J$, and formally substituting

$$
\begin{aligned}
& \tilde{U}(p) \sim \varepsilon^{2} \tilde{U}^{2}(p)+\varepsilon^{3} \tilde{U}^{3}(p)+\varepsilon^{4} \tilde{U}^{4}(p)+\cdots, \\
& \tilde{\Xi}(p) \sim \varepsilon^{2} \tilde{\Xi}^{2}(p)+\varepsilon^{3} \tilde{\Xi}^{3}(p)+\varepsilon^{4} \underline{\Xi}^{4}(p)+\cdots,
\end{aligned}
$$


we arrive at the following sequence of problems, parametrized by $\theta \in \mathbb{R} / L$ and defined in the semi-infinite strip $\Sigma$ :

$$
\begin{gathered}
\int_{\Sigma} \tilde{\Xi}_{n}^{k}(p) \tilde{\tau}_{n}+\tilde{\Xi}_{3}^{k}(p) \tilde{\tau}_{3}+\tilde{U}^{k}(p)\left(\partial_{\tilde{\rho}} \tilde{\tau}_{n}+\partial_{3} \tilde{\tau}_{3}\right) d \tilde{\rho} d x_{3} \\
=-\int_{-1}^{1} u^{k}(p)\left(0, \theta, x_{3}\right) \tilde{\tau}_{n}\left(0, x_{3}\right) d x_{3} \quad \text { for all } \underset{\sim}{\tau} \in \underset{\sim}{S_{0}(\Sigma, p),} \\
\int_{\Sigma}\left[\partial_{\tilde{\rho}} \tilde{\Xi}_{n}^{k}(p)+\partial_{3} \tilde{\Xi}_{3}^{k}(p)\right] v d \tilde{\rho} d x_{3}=\int_{\Sigma} G_{k}(p) v d \tilde{\rho} d x_{3} \quad \text { for all } v \in V(\Sigma, p), \\
\tilde{\Xi}_{s}^{k}(p)=\tilde{\rho} \kappa(\theta) \tilde{\Xi}_{s}^{k-1}(p)+\partial_{\theta} \tilde{U}^{k}(p), \\
G_{k}(p)=\sum_{j=0}^{k-2} \tilde{\rho}^{j}\left(a_{1}^{j} \tilde{\Xi}_{n}^{k-j-1}(p)+a_{2}^{j} \partial_{\theta \theta} \tilde{U}^{k-j-2}(p)+a_{3}^{j} \partial_{\theta} \tilde{U}^{k-j-2}(p)\right),
\end{gathered}
$$

where $u^{k}(p)=0$ for $k$ odd and

$$
\begin{aligned}
& V(\Sigma, p)=\left\{v \in \mathcal{D}^{\prime}(\Sigma):(1+\tilde{\rho})^{-1} v \in L^{2}(\Sigma), \operatorname{deg}_{3} v \leq p\right\}, \\
& \underset{\sim}{S_{0}}(\Sigma, p)=\left\{\underset{\sim}{\tau} \in{\underset{\sim}{\mathcal{D}}}^{\prime}(\Sigma):(1+\tilde{\rho}) \operatorname{div} \underset{\sim}{\tau} \in L^{2}(\Sigma), \underset{\sim}{\tau} \in \underset{\sim}{L^{2}}(\Sigma),\right. \\
& \left.\operatorname{deg}_{3} \underset{\sim}{\sim} \leq p, \operatorname{deg}_{3} \tau_{3} \leq p+1\right\} .
\end{aligned}
$$

These are appropriate spaces to pose the boundary corrector problem. Looking at (2.4), note that $\tilde{U}^{k}(p)$ and $\tilde{\Xi}^{\tilde{\Xi}}(p)$ are mixed approximations for $\tilde{U}^{k}$ and $\nabla \tilde{U}^{k}$.

We show below in Lemma 2.2 that (2.17) is well-posed, and that the solutions $\tilde{U}^{k}$ and $\tilde{\Xi}^{k}$ decay exponentially to zero with $\tilde{\rho}$.

Finally, the following expansions hold:

$$
\begin{aligned}
u^{\varepsilon}(p)\left(\underline{x}^{\varepsilon}\right) \sim & \sum_{k \geq 0} \varepsilon^{2 k} u^{2 k}(p)\left({\underset{\sim}{x}}^{\varepsilon}, \varepsilon^{-1} x_{3}^{\varepsilon}\right)-\chi(\rho) \sum_{k \geq 2} \varepsilon^{k} \tilde{U}^{k}(p)\left(\varepsilon^{-1} \rho, \theta, \varepsilon^{-1} x_{3}^{\varepsilon}\right), \\
\stackrel{\sigma}{\sim}^{\varepsilon}(p)\left(\underline{x}^{\varepsilon}\right) \sim & \sum_{k \geq 0} \varepsilon^{2 k}\left(\begin{array}{c}
{\underset{\sigma}{\sim}}^{2 k}(p) \\
\varepsilon^{-1} \sigma_{3}^{2 k}(p)
\end{array}\right)\left({\underset{\sim}{x}}^{\varepsilon}, \varepsilon^{-1} x_{3}^{\varepsilon}\right) \\
& -\chi(\rho) \sum_{k \geq 2} \varepsilon^{k} \underline{\tilde{\Xi}}^{k}(p)\left(\varepsilon^{-1} \rho, \theta, \varepsilon^{-1} x_{3}^{\varepsilon}\right),
\end{aligned}
$$

where

$$
\underline{\Xi}^{k}(p)=\left(\varepsilon^{-1} \tilde{\Xi}_{n}^{k}(p) \underset{\sim}{n}+\tilde{\Xi}_{s}^{k}(p) \underset{\sim}{s}, \varepsilon^{-1} \Xi_{3}^{k}(p)\right) .
$$

The above formal reasoning shall be justified in Theorem 2.2. First we study the terms present in the expansion.

We present below the stability result regarding the boundary correctors for the models. The existence, uniqueness and the first inequality in (2.20) follows from standard theory for mixed problems. ${ }^{8,19}$ The exponential decay is an application of Theorem 5.4 of Arnold and Madureira. ${ }^{3}$

Lemma 2.2. Assume, for a fixed positive integer $k$, that $u^{k}$ is defined as above. Then, for each $\theta$, there exists a unique solution $\tilde{U}^{k}(p) \in V(\Sigma, p)$, and 
$\left(\tilde{\Xi}_{n}^{k}(p), \tilde{\Xi}_{3}^{k}(p)\right) \in \underset{\sim}{S_{0}}(\Sigma, p)$ to (2.17). Also, there exist positive constants $C$ and $\alpha$ such that

$$
\begin{aligned}
& \left\|(1+\tilde{\rho})^{-1} \tilde{U}^{k}(p)\right\|_{L^{2}(\Sigma)}+\left\|\left|\tilde{\Xi}_{n}^{k}(p)\right|+\left|\tilde{\Xi}_{3}^{k}(p)\right|\right\|_{L^{2}(\Sigma)} \\
& +\left\|(1+\tilde{\rho})\left[\partial_{\tilde{\rho}} \tilde{\Xi}_{n}^{k}(p)+\partial_{3} \tilde{\Xi}_{3}^{k}(p)\right]\right\|_{L^{2}(\Sigma)} \leq C, \\
& \int_{t}^{\infty} \int_{-1}^{1}\left[\tilde{U}^{k}(p)\right]^{2}+{\left.\underset{\sim}{\tilde{\Xi}^{k}}(p)\right|^{2} d x_{3} d \tilde{\rho} \leq C e^{-\alpha t},}
\end{aligned}
$$

for every non-negative real number $t$ and every positive integer $p$. The constant $\alpha$ may depend on $\Omega$ and $k$, but is independent of $f, g$ and $p$, while the constant $C$ may depend on $\Omega, k, f$ and $g$.

In the remainder of this section, we estimate the convergence rates of the truncated asymptotic expansions.

Theorem 2.2. For each positive integer $N$, let

$$
\begin{aligned}
e_{2 N}(p)\left(\underline{x}^{\varepsilon}\right)= & u^{\varepsilon}(p)\left(\underline{x}^{\varepsilon}\right)-\sum_{k=0}^{N} \varepsilon^{2 k} u^{2 k}(p)\left({\underset{\sim}{\sim}}^{\varepsilon}, \varepsilon^{-1} x_{3}\right) \\
& +\chi(\rho) \sum_{k=1}^{2 N} \varepsilon^{k} \tilde{U}^{k}(p)\left(\varepsilon^{-1} \rho, \theta, \varepsilon^{-1} x_{3}\right), \\
\underline{\Delta}_{2 N}(p)\left(\underline{x}^{\varepsilon}\right)= & \underline{\sigma}^{\varepsilon}(p)\left(\underline{x}^{\varepsilon}\right)-\sum_{k=0}^{N} \varepsilon^{2 k}\left(\begin{array}{c}
\underset{\sigma^{2 k}}{\sim}(p) \\
\varepsilon^{-1} \sigma_{3}^{2 k}(p)
\end{array}\right)\left(\underline{\sim}_{\sim}^{\varepsilon}, \varepsilon^{-1} x_{3}\right) \\
& +\chi(\rho) \sum_{k=2}^{2 N} \varepsilon^{k}\left(\begin{array}{c}
\varepsilon^{-1} \tilde{\Xi}_{n}^{k}(p) \underset{\sim}{\sim}+\tilde{\Xi}_{s}^{k}(p) \underset{\sim}{s} \\
\varepsilon^{-1} \Xi_{3}^{k}(p)
\end{array}\right)\left(\varepsilon^{-1} \rho, \theta, \varepsilon^{-1} x_{3}\right) .
\end{aligned}
$$

Then there exists a constant $C$ such that

$$
\left\|e_{2 N}(p)\right\|_{L^{2}\left(P^{\varepsilon}\right)}+\left\|\underline{\Delta}_{2 N}(p)\right\|_{\underline{H}\left(\operatorname{div}, P^{\varepsilon}\right)} \leq C \varepsilon^{2 N+1} .
$$

Note that the above convergence in $\varepsilon$ is the same as in Theorem 2.1. The proof of Theorem 2.2 uses the theory of mixed problems, ${ }^{8}$ and we shall go through the main steps.

In what follows, we denote by $\pi_{p}$ the orthogonal projection from $L^{2}(-1,1)$ to $\mathbb{P}_{p}(-1,1)$, and by $\stackrel{\circ}{\pi}^{1}$ the orthogonal projection operator from $\stackrel{\circ}{H}^{1}(-1,1)$ to $\stackrel{\circ}{\mathbb{P}}_{p}(-1,1)$, with respect to the inner product that induces the norm $|\cdot|_{H^{1}(-1,1)}$. We need the following technical result.

Lemma 2.3. If $\tau \in \stackrel{\circ}{H}^{1}(-1,1)$, then $\left(\stackrel{\circ}{\pi}_{p+1}^{1} \tau\right)^{\prime}=\pi_{p} \tau^{\prime}$ and if $\phi \in H^{1}(-1,1) \cap$ $\hat{L}^{2}(-1,1)$, then $\left(\hat{\pi}_{p}^{1} \phi\right)^{\prime}=\pi_{p-1} \phi^{\prime}$. 
Proof. For any $v \in \mathbb{P}_{p}(-1,1)$, we can write $v=\stackrel{\circ}{v}^{\prime}+c$, where $\stackrel{\circ}{v}\left(\tilde{\rho}_{2}\right)=$ $\int_{-1}^{\tilde{\rho}_{2}} v(s)-c d s$ and $c=(1 / 2) \int_{-1}^{1} v(s) d s$. Note that $\stackrel{\circ}{v} \in \stackrel{\circ}{H}^{1}(-1,1)$ and for $\tau \in \stackrel{\circ}{H}^{1}(-1,1)$,

$$
\int_{-1}^{1}\left(\stackrel{\circ}{\pi}_{p+1}^{1} \tau\right)^{\prime} v d \tilde{\rho}_{2}=\int_{-1}^{1}\left(\stackrel{\circ}{\pi}_{p+1}^{1} \tau\right)^{\prime}\left(\stackrel{\circ}{v}^{\prime}+c\right) d \tilde{\rho}_{2}=\int_{-1}^{1} \tau^{\prime}\left(\stackrel{\circ}{v}^{\prime}+c\right) d \tilde{\rho}_{2}=\int_{-1}^{1} \tau^{\prime} v d \tilde{\rho}_{2} .
$$

So $\left(\stackrel{\circ}{p+1}_{p+1}^{1} \tau\right)^{\prime}=\pi_{p} \tau^{\prime}$. The second identity of the lemma follows from similar arguments.

We denote the orthogonal $L^{2}$ projection in the vertical direction by $\pi_{p}^{\left(x_{3}\right)}$, i.e. if $v \in L^{2}(P)$, then $\pi_{p}^{\left(x_{3}\right)} v \in L^{2}\left(\Omega ; \mathbb{P}_{p}(-1,1)\right)$ is such that

$$
\int_{P}\left(\pi_{p}^{\left(x_{3}\right)} v-v\right) \psi d \underline{x}=0 \quad \text { for all } \psi \in L^{2}\left(\Omega ; \mathbb{P}_{p}(-1,1)\right) .
$$

Similar notation holds for $\stackrel{\circ}{p}_{p}^{\left(x_{3}\right)}$.

Before proving the main result, we show how the solutions of a mixed, $\varepsilon$-dependent problem in $P$ behave. Recall that we define $V(P, p)$ and $\underline{S}_{0}(P, p)$ in $(2.6)$.

Theorem 2.3. Let $\tilde{F} \in\left(\underline{S}_{0}(P, p)\right)^{*}$, the dual space of $\underline{S}_{0}(P, p)$, and let $\tilde{g} \in L^{2}(P)$. Then there exists unique $u \in V(P, p)$ and $\sigma \in \underline{S}_{0}(P, p)$ such that

$$
\begin{array}{r}
\int_{P}\left(\underset{\sim}{\sigma} \cdot \underset{\sim}{\tau}+\varepsilon^{-2} \sigma_{3} \tau_{3}\right) d \underline{x}+\int_{P} u\left(\operatorname{div} \underset{\sim}{\tau}+\varepsilon^{-2} \partial_{3} \tau_{3}\right) d \underline{x}=\tilde{F}(\underset{\sim}{\tau}) \\
\text { for all } \underline{\tau} \in \underline{S}_{0}(P, p), \\
\int_{P}\left(\operatorname{div} \underset{\sim}{\sigma}+\varepsilon^{-2} \partial_{3} \sigma_{3}\right) v d \underline{x}=\int_{P} \tilde{g} v d \underline{x} \quad \text { for all } v \in V(P, p) .
\end{array}
$$

Moreover, there is a universal constant $c$ such that

$$
\begin{aligned}
& \|u\|_{L^{2}(P)}+\|\underset{\sim}{\sigma}\|_{L^{2}(P)}+\varepsilon^{-1}\left\|\sigma_{3}\right\|_{L^{2}(P)}+\left\|\operatorname{div} \underset{\sim}{\sigma}+\varepsilon^{2} \partial_{3} \sigma_{3}\right\|_{L^{2}(P)} \\
& \quad \leq c\left(\|\tilde{F}\|_{\left(\underline{S}_{0}(P, p)\right) *}+\|\tilde{g}\|_{L^{2}(P)}\right) .
\end{aligned}
$$

Proof. Let

$$
\begin{aligned}
& M=V(P, p), \quad\|v\|_{M}=\|v\|_{L^{2}(P)}, \\
& X=\underline{S}_{0}(P, p), \quad\|\underline{\tau}\|_{X}^{2}=\|\underset{\sim}{\tau}\|_{L^{2}(P)}^{2}+\varepsilon^{-2}\left\|\tau_{3}\right\|_{L^{2}(P)}^{2}+\left\|\operatorname{div} \underset{\sim}{\tau}+\varepsilon^{-2} \partial_{3} \tau_{3}\right\|_{L^{2}(P)}^{2}, \\
& a(\underline{\sigma}, \underline{\tau})=\int_{P} \underset{\sim}{\sigma} \cdot \underset{\sim}{\tau}+\varepsilon^{-2} \sigma_{3} \tau_{3} d \underline{x}, \quad b(\underline{\tau}, v)=\int_{P}\left(\operatorname{div} \underset{\sim}{\tau}+\varepsilon^{-2} \partial_{3} \tau_{3}\right) v d \underline{x} .
\end{aligned}
$$

Since $\operatorname{div} \underset{\sim}{\tau}+\varepsilon^{-2} \partial_{3} \tau_{3} \in V(P, p)$ for all $\underline{\tau} \in \underline{S}_{0}(P, p)$, the coercivity hypothesis

$$
a(r, r) \geq\|r\|_{X}^{2} \quad \text { for all } r \in\{\tilde{r} \in X: b(\tilde{r}, w)=0 \text {, for all } w \in M\}
$$


holds immediately. Now we want to show that the inf-sup hypothesis

$$
\sup _{r \in X} \frac{b(r, v)}{\|r\|_{X}} \geq k_{0}\|v\|_{M} \quad \text { for all } v \in M
$$

is also satisfied for some positive constant $k_{0}$. Let $v \in V(P, p)$, and define $V(P)=$ $\left\{\tilde{v} \in H^{1}(P): \tilde{v}=0\right.$ on $\left.\partial P_{L}\right\}$, and $u \in V(P)$ such that

$$
\int_{P} \underset{\sim}{\nabla} u \cdot \underset{\sim}{\nabla} \tilde{v}+\varepsilon^{-2} \partial_{3} u \partial_{3} \tilde{v}=\int_{P} v \tilde{v} d \underline{x} \quad \text { for all } \tilde{v} \in V(P) .
$$

Then $\|u\|_{H^{1}(P)} \leq c\|v\|_{L^{2}(P)}$, where $c$ is a universal constant. Moreover,

$$
\begin{aligned}
\int_{\Omega} \varepsilon^{-2}|u(\underset{\sim}{x}, \cdot)|_{H^{1}(-1,1)}^{2} d \underset{\sim}{x} & =\int_{P} \varepsilon^{-2}\left[\partial_{3} u\left(\underset{\sim}{x}, x_{3}\right)\right]^{2} d \underline{x} \\
& \leq \int_{P}\left|\underset{\sim}{\nabla} u\left(\underset{\sim}{x}, x_{3}\right)\right|^{2}+\varepsilon^{-2}\left[\partial_{3} u\left(\underset{\sim}{x}, x_{3}\right)\right]^{2} d \underline{x} \\
& =\int_{P} v u d \underline{x} \leq\|v\|_{L^{2}(P)}\|u\|_{L^{2}(P)} \\
& \leq c\|v\|_{L^{2}(P)}^{2} .
\end{aligned}
$$

Set $\underset{\sim}{\sigma}=\underset{\sim}{\nabla} u, \sigma_{3}=\partial_{3} u$ and $\underset{\sim}{\sigma}=\left(\underset{\sim}{\sigma}, \sigma_{3}\right)$. We cannot use $\underset{\sim}{\sigma}$ as a "candidate" for the inf-sup condition since $\underset{\sim}{\sigma}$ does not belong to $\underline{S}_{0}(P, p)$ in general. Let $\hat{u}\left(\underset{\sim}{x}, x_{3}\right)=$ $u\left(\underset{\sim}{x}, x_{3}\right)-(1 / 2) \int_{-1}^{1} u\left(\underset{\sim}{x}, x_{3}\right) d x_{3}$, and for each $\underset{\sim}{x} \in \Omega$, define $\sigma_{3}(p)(\underset{\sim}{x}, \cdot) \in \stackrel{\stackrel{\circ}{\mathbb{P}}}{p+1}$ $(-1,1)$ and $u(p)(\underset{\sim}{x}, \cdot) \in \hat{\mathbb{P}}_{p}(-1,1)$ such that

$$
\begin{array}{ll}
\int_{-1}^{1}\left[\sigma_{3}\left(\underset{\sim}{x}, x_{3}\right)-\sigma_{3}(p)\left(\underset{\sim}{x}, x_{3}\right)\right] \tau\left(x_{3}\right)+\left[\hat{u}\left(\underset{\sim}{x}, x_{3}\right)-u(p)\left(\underset{\sim}{x}, x_{3}\right)\right] \partial_{3} \tau\left(x_{3}\right) d x_{3}=0 \\
& \text { for all } \tau \in \mathbb{P}_{p+1}(-1,1), \\
\int_{-1}^{1} \partial_{3}\left[\sigma_{3}\left(\underset{\sim}{x}, x_{3}\right)-\sigma_{3}(p)\left(\underset{\sim}{x}, x_{3}\right)\right] \hat{v}\left(x_{3}\right) d x_{3}=0 & \text { for all } \hat{v} \in \hat{\mathbb{P}}_{p}(-1,1) .
\end{array}
$$

Then $\partial_{3} \sigma_{3}(p)=\pi_{p}^{\left(x_{3}\right)} \partial_{3} \sigma_{3}$, and using Lemma 2.3 we conclude that $\sigma_{3}(p)=\stackrel{\circ}{\pi}_{p+1}^{\left(x_{3}\right)} \sigma_{3}$. It follows that

$$
\begin{aligned}
\varepsilon^{-2}\left\|\sigma_{3}(p)\right\|_{L^{2}(P)}^{2} & \left.=\int_{-1}^{1} \varepsilon^{-2} \| \sigma_{3}(p) \underset{\sim}{x}, \cdot\right) \|_{L^{2}(-1,1)}^{2} d \underset{\sim}{x} \\
& \leq c \int_{-1}^{1} \varepsilon^{-2}\left\|\sigma_{3}(\underset{\sim}{x}, \cdot)\right\|_{L^{2}(-1,1)}^{2} d \underset{\sim}{x} \\
& =c \int_{-1}^{1} \varepsilon^{-2}|u(\underset{\sim}{x}, \cdot)|_{H^{1}(-1,1)}^{2} d \underset{\sim}{x} \leq c\|v\|_{L^{2}(P)}^{2} .
\end{aligned}
$$

Define now $\underset{\sim}{\sigma}(p)=\pi_{p}^{\left(x_{3}\right)} \underset{\sim}{\sigma}$. Then

$$
\begin{aligned}
& \|\underset{\sim}{\sigma}(p)\|_{L^{2}(P)} \leq c\|\underset{\sim}{\sigma}\|_{L^{2}(P)} \leq c\|v\|_{L^{2}(P)}, \\
& \left\|\operatorname{div} \underset{\sim}{\sigma}(p)+\varepsilon^{-2} \partial_{3} \sigma_{3}(p)\right\|_{L^{2}(P)}=\left\|\pi_{p}^{\left(x_{3}\right)} \operatorname{div} \underset{\sim}{\sigma}+\varepsilon^{-2} \pi_{p}^{\left(x_{3}\right)} \partial_{3} \sigma_{3}\right\|_{L^{2}(P)} \\
& \leq c\left\|\operatorname{div} \underset{\sim}{\sigma}+\varepsilon^{-2} \partial_{3} \sigma_{3}\right\|_{L^{2}(P)}=c\|v\|_{L^{2}(P)} .
\end{aligned}
$$


Thus, from (2.26) and (2.27), $\|\sigma(p)\|_{X} \leq c\|v\|_{L^{2}(P)}$. We can finally prove the inf-sup condition, since for all $v \in V(\widetilde{P} ; p)$,

$$
\begin{aligned}
\sup _{\underset{\sim}{\tau} \in X} \frac{1}{\|\underline{\tau}\|_{X}} \int_{P} v\left(\operatorname{div} \underset{\sim}{\tau}+\varepsilon^{-2} \partial_{3} \tau_{3}\right) d \underline{x} & \geq \frac{1}{\|\underline{\sigma}(p)\|_{X}} \int_{P} v\left[\operatorname{div} \underset{\sim}{\sigma}(p)+\varepsilon^{-2} \partial_{3} \sigma_{3}(p)\right] d \underline{x} \\
& =\frac{1}{\|\underline{\sigma}(p)\|_{X}} \int_{P} v\left[\pi_{p}^{\left(x_{3}\right)} \operatorname{div} \underset{\sim}{\sigma}+\varepsilon^{-2} \pi_{p}^{\left(x_{3}\right)} \partial_{3} \sigma_{3}\right] d \underline{x} \\
& =\frac{1}{\|\underline{\sigma}(p)\|_{X}} \int_{P} v\left(\operatorname{div} \underset{\sim}{\sigma}+\varepsilon^{-2} \partial_{3} \sigma_{3}\right) d \underline{x} \\
& =\frac{\|v\|_{L^{2}(P)}^{2}}{\|\underline{\sigma}(p)\|_{X}} \geq c\|v\|_{L^{2}(P)},
\end{aligned}
$$

and (2.24) follows. Thus we conclude the present result.

We need the following notation in the proof of the next result. Define

$$
\begin{aligned}
& u_{2 N}(p)(\underline{x})=\sum_{k=0}^{N} \varepsilon^{2 k} u^{2 k}(p)(\underline{x}), \quad \underline{\sigma}_{2 N}(p)(\underline{x})=\sum_{k=0}^{N} \varepsilon^{2 k} \underline{\sigma}^{2 k}(p)(\underline{x}), \\
& U_{2 N}(p)(\underline{x})=\chi(\rho) \sum_{k=2}^{2 N} \varepsilon^{k} \tilde{U}^{k}(p)\left(\varepsilon^{-1} \rho, \theta, x_{3}\right), \\
& \underline{\Xi}_{2 N}(p)(\underline{x})=\chi(\rho) \sum_{k=2}^{2 N} \varepsilon^{k}\left(\begin{array}{c}
\varepsilon^{-1} \tilde{\Xi}_{n}^{\tilde{\Xi}_{n}}(p) \underset{\sim}{\tilde{\sim}}+\tilde{\Xi}_{s}^{k}(p) \underset{\sim}{s} \\
\varepsilon^{-1} \underline{\Xi}_{3}^{k}(p)
\end{array}\right)\left(\varepsilon^{-1} \rho, \theta, x_{3}\right) .
\end{aligned}
$$

Proof. (of Theorem 2.2) For all $\tau \in S_{0}(P, p)$, define $\tilde{\tau}_{s}$ as in (2.15), (2.16). Then it follows from the construction of the terms in the asymptotic expansion, and Lemma 2.2 that

$$
\begin{aligned}
& \int_{P}\left[\underset{\sim}{\sigma}(p)-{\underset{\sim}{\sim}}_{2 N}(p)+{\underset{\sim}{\Xi}}_{2 N}(p)\right] \cdot \underset{\sim}{\tau}+\varepsilon^{-2}\left[\sigma_{3}(p)-\left(\sigma_{2 N}\right)_{3}(p)+\left(\Xi_{2 N}\right)_{3}(p)\right] \tau_{3} d \underline{x} \\
& \quad+\int_{P}\left[u(p)-u_{2 N}(p)+U_{2 N}(p)\right]\left(\operatorname{div} \underset{\sim}{\tau}+\varepsilon^{-2} \partial_{3} \tau_{3}\right) d \underline{x}=R_{1}\left(N, \tau_{s}\right), \\
& \int_{P}\left\{\operatorname{div}\left[\underset{\sim}{\sigma}(p)-{\underset{\sim}{\sigma}}_{2 N}(p)+{\underset{\sim}{\Xi}}_{2 N}(p)\right]+\varepsilon^{-2} \partial_{3}\left[\sigma_{3}(p)-\left(\sigma_{2 N}\right)_{3}(p)+\left({\underset{\sim}{\Xi}}_{2 N}\right)_{3}(p)\right]\right\} v d \underline{x} \\
& \quad=R_{2}(N, v),
\end{aligned}
$$

where $\left|R_{1}\left(N, \tau_{s}\right)\right| \leq C \varepsilon^{2 N}\|\underline{\tau}\|_{S_{0}(P, p)}$, and $\left|R_{2}(N, v)\right| \leq C \varepsilon^{2 N}\|v\|_{V(P, p)}$. Using Lemma 2.3, we have that

$$
\begin{aligned}
& \left\|u(p)-u_{2 N}(p)+U_{2 N}\right\|_{L^{2}(P)}+\left\|\underset{\sim}{\sigma}(p)-\underset{\sim}{\sigma_{2 N}}(p)+{\underset{\sim}{\widetilde{\Xi}}}_{2 N}(p)\right\|_{L^{2}(P)} \\
& \quad+\varepsilon^{-1} \| \sigma_{3}(p)-\left(\sigma_{2 N}\right)_{3}(p)+\left(\underset{\tilde{\Xi}_{2 N}}{\sigma_{3}}(p) \|_{L^{2}(P)}\right. \\
& \quad+\left\|\operatorname{div}\left[\underset{\sim}{\sigma}(p)-{\underset{\sim}{\sigma}}_{2 N}(p)+{\underset{\sim}{\sim}}_{2 N}(p)\right]+\varepsilon^{-2} \partial_{3}\left[\sigma_{3}(p)-\left(\sigma_{2 N}\right)_{3}(p)+\left(\Xi_{2 N}\right)_{3}(p)\right]\right\|_{L^{2}(P)} \\
& \leq C \varepsilon^{2 N} .
\end{aligned}
$$


Next, to conclude the final result, we add and subtract new terms, using the triangle inequality, estimate (2.28), and Lemma 2.2, and finally scaling the domain, from $P$ to $P^{\varepsilon}$.

\section{Estimates for the Modeling Error}

We begin now the central part of this paper, the derivation of error bounds for the models $\mathrm{SP}_{2}^{\prime}$. An essential part is to estimate the difference between individual terms in the asymptotic expansions.

We need the following technical result. ${ }^{19}$

Lemma 3.1. Given $u \in H^{2}(-1,1) \cap \hat{L}^{2}(-1,1)$ and $\sigma=u^{\prime}$, there exists unique $u(p) \in \hat{\mathbb{P}}_{p}(-1,1)$ and $\sigma(p) \in \mathbb{P}_{p+1}(-1,1)$ with $\sigma(p)(-1)=\sigma(-1)$, and $\sigma(p)(1)=$ $\sigma(1)$, such that

$$
\begin{aligned}
& \int_{-1}^{1}[\sigma-\sigma(p)] \tau+[u-u(p)] \tau^{\prime} d x=0 \quad \text { for all } \tau \in \stackrel{\circ}{\mathbb{P}}_{p+1}(-1,1), \\
& \int_{-1}^{1}[\sigma-\sigma(p)]^{\prime} v d x_{2}=0 \quad \text { for all } v \in \hat{\mathbb{P}}_{p}(-1,1) .
\end{aligned}
$$

Moreover, for any non-negative real number $s$, there exists a constant $C$ such that

$$
\begin{aligned}
\|u-u(p)\|_{L^{2}(-1,1)} & \leq C p^{-2-s}\|u\|_{H^{s+2}(-1,1)}, \\
\|u-u(p)\|_{H^{1 / 2}(-1,1)} & \leq C p^{-1-s}\|u\|_{H^{s+2}(-1,1)}, \\
\|\sigma-\sigma(p)\|_{L^{2}(-1,1)} & \leq C p^{-1-s}\|u\|_{H^{s+2}(-1,1)}, \\
\|\sigma-\sigma(p)\|_{H^{1}(-1,1)} & \leq C p^{-s}\|u\|_{H^{s+2}(-1,1)} .
\end{aligned}
$$

In what follows, we need the definitions below.

Definition 3.1. For a non-negative real number $s$, let

$$
\begin{gathered}
a_{s}=\|f\|_{L^{2}\left(\Omega ; H^{s}(-1,1)\right)}+\|g\|_{L^{2}\left(\partial P_{ \pm}\right)}, \quad a_{s}^{1}=\|f\|_{H^{1}\left(\Omega ; H^{s}(-1,1)\right)}+\|g\|_{H^{1}\left(\partial P_{ \pm}\right)}, \\
a_{s}^{b}=\left(\int_{\partial \Omega}\|f(\underset{\sim}{x}, \cdot)\|_{H^{s}(-1,1)}^{2}+|g(\underset{\sim}{x},-1)|^{2}+|g(\underset{\sim}{x}, 1)|^{2} d \underset{\sim}{x}\right)^{1 / 2} .
\end{gathered}
$$

We note from Lemma 2.1 that there exists a constant $c$ independent of $f$ and $g$ such that

$$
\begin{aligned}
\left\|u^{2}\right\|_{L^{2}\left(\Omega ; H^{s}(-1,1)\right)} & \leq c a_{s}, \\
\left\|\underset{\sim}{\nabla} u^{2}\right\|_{L^{2}\left(\Omega ; H^{s}(-1,1)\right)} & \leq c a_{s}^{1}, \\
\left\|u^{2}\right\|_{L^{2}\left(\partial \Omega ; H^{s}(-1,1)\right)} & \leq c a_{s}^{b} .
\end{aligned}
$$

To simplify the notation, henceforward we denote $\sigma_{3}^{2}(\underline{x})=\partial_{x_{3}} u^{2}(\underline{x})$. 
Lemma 3.2. Assume that $u^{2}, \sigma_{3}^{2}, u^{2}(p), \sigma_{3}^{2}(p)$ are defined as above. Then, with $\underset{\sim}{x} \in \Omega$ as a parameter,

$$
\begin{array}{ll}
\int_{-1}^{1}\left[\sigma_{3}^{2}\left(\underset{\sim}{x}, x_{3}\right)-\sigma_{3}^{2}(p)\left(\underset{\sim}{x}, x_{3}\right)\right] \tau\left(x_{3}\right)+\left[u_{\left.\underset{\sim}{2}\left(\underset{x}{x}, x_{3}\right)-u^{2}(p)\left(\underset{\sim}{x}, x_{3}\right)\right] \tau^{\prime}\left(x_{3}\right) d x_{3}=0}\right. & \text { for all } \tau \in \mathbb{\stackrel { \circ } { P }}_{p+1}(-1,1), \\
\left.\left.\int_{-1}^{1} \partial_{3}\left[\sigma_{3}^{2} \underset{\sim}{x}, x_{3}\right)-\sigma_{3}^{2}(p) \underset{\sim}{x}, x_{3}\right)\right] v\left(x_{3}\right) d x_{3}=0 & \text { for all } v \in \hat{\mathbb{P}}_{p}(-1,1) .
\end{array}
$$

Moreover, for any non-negative real number $s$, there exists a constant $C$ such that

$$
\begin{aligned}
\left\|u^{2}-u^{2}(p)\right\|_{L^{2}(P)} & \leq C p^{-2-s} a_{s}, \\
\left\|\underset{\sim}{\nabla} u^{2}-\underset{\sim}{\nabla} u^{2}(p)\right\|_{L^{2}(P)} & \leq C p^{-2-s} a_{s}^{1}, \\
\left\|\sigma_{3}^{2}-\sigma_{3}^{2}(p)\right\|_{L^{2}(P)} & \leq C p^{-1-s} a_{s}, \\
\left\|\partial_{x_{3}^{\varepsilon}} \sigma_{3}^{2}-\partial_{x_{3}^{\varepsilon}} \sigma_{3}^{2}(p)\right\|_{L^{2}(P)} & \leq C p^{-s} a_{s} .
\end{aligned}
$$

Proof. The first part of the lemma results from (2.2), (2.11). To obtain the estimates (3.4), it is enough to apply Lemma 3.1, in each vertical fiber, and then integrate in $\Omega$, and use (3.1) and (3.2).

We estimate now the error due to the first boundary layer terms. The following definition is useful.

Definition 3.2. Let $\underset{\sim}{x} \in \partial \Omega$ and $s$ be a non-negative real number. Let

$$
N(s)=\max \{n \in \mathbb{Z}: 2 n<s\} .
$$

If $\sup _{x_{3} \in\{-1,1\}}\left|g\left(\underset{\sim}{x}, x_{3}\right)\right| \neq 0$, set $m=1$. If $|g(\underset{\sim}{x},-1)|=|g(\underset{\sim}{x}, 1)|=0$ and

$$
\sup _{x_{3} \in\{-1,1\}} \sum_{j=2}^{N(s+5 / 2)}\left|\partial_{3}^{2 j-3} f\left(\underset{\sim}{x}, x_{3}\right)\right| \neq 0,
$$

let $m$ be the minimum integer in $\{2, \ldots, N(s+5 / 2)\}$ such that

$$
\sup _{x_{3} \in\{-1,1\}}\left|\partial_{3}^{2 m-3} f\left(\underset{\sim}{x}, x_{3}\right)\right| \neq 0 .
$$

We define in both cases $\mu(\underset{\sim}{x}, s, \delta)=\min \{4 m-3-\delta, s+3 / 2\}$. If $\mid g(\underset{\sim}{(x,-1)} \mid=$ $|g(\underset{\sim}{x}, 1)|=0$ and $(3.6)$ does not hold, then define $\mu(\underset{\sim}{x}, s, \delta)=s+3 / 2$. Finally, set

$$
\bar{\mu}(s, \delta)=\inf _{\sim} \in \partial \Omega(\underset{\sim}{x}, s, \delta) .
$$

The next result estimates the boundary correctors in $P$. We present its proof in the Appendix. Define

$$
\tilde{\Xi}_{n}^{2}=\partial_{\tilde{\rho}} \tilde{U}^{2}, \quad \tilde{\Xi}_{3}=\partial_{x_{3}} \tilde{U}^{2} .
$$


Lemma 3.3. Let

$$
\begin{aligned}
& \Upsilon_{n}\left(\underline{x}^{\varepsilon}\right)=\chi(\rho)\left[\tilde{\Xi}_{n}^{2}-\tilde{\Xi}_{n}^{2}(p)\right]\left(\varepsilon^{-1} \rho, \theta, \varepsilon^{-1} x_{3}^{\varepsilon}\right), \\
& \Upsilon_{3}\left(\underline{x}^{\varepsilon}\right)=\chi(\rho)\left[\tilde{\Xi}_{3}^{2}-\tilde{\Xi}_{3}^{2}(p)\right]\left(\varepsilon^{-1} \rho, \theta, \varepsilon^{-1} x_{3}^{\varepsilon}\right) .
\end{aligned}
$$

For any non-negative real number $s$ such that $s+1 / 2$ is not an even integer, and for any arbitrarily small $\delta>0$, there exists a constant $c$ such that

$$
\left\|\Upsilon_{n}\right\|_{L^{2}\left(P^{\varepsilon}\right)}+\left\|\Upsilon_{3}\right\|_{L^{2}\left(P^{\varepsilon}\right)} \leq c \varepsilon\left(p^{-1-s}+p^{-\bar{\mu}(s, \delta)}\right) a_{s}^{b} .
$$

We are ready to estimate the difference between the exact and model solutions in several norms. This is the main result of the paper. To also consider interior estimates, which disregard the boundary layer, define $P_{0}^{\varepsilon}=\Omega_{0} \times(-\varepsilon, \varepsilon)$, where $\Omega_{0}$ is an open domain such that $\bar{\Omega}_{0} \subset \Omega$. We present interior estimates only when these have better rates of convergence than global estimates.

Theorem 3.1. For any non-negative real numbers $s$ and $s^{*}$ such that $s^{*}+1 / 2$ is not an even integer, and for any arbitrarily small $\delta>0$, there exist constants $c$ and $C$ independent of $\varepsilon$ and $p$, with $c$ also independent of $f$ and $g$, such that the error between $u^{\varepsilon}$ and its approximation $u^{\varepsilon}(p)$ is bounded as

$$
\begin{aligned}
\left\|u^{\varepsilon}-u^{\varepsilon}(p)\right\|_{L^{2}\left(P^{\varepsilon}\right)} & \leq C \varepsilon^{5 / 2} p^{-2-s} a_{s}+C \varepsilon^{3}, \\
\left\|\sigma_{\sim}^{\varepsilon} \cdot \underset{\sim}{\sim}-\underset{\sim}{\sigma^{\varepsilon}}(p) \cdot \underset{\sim}{n}\right\|_{L^{2}\left(P^{\varepsilon}\right)} & \leq C \varepsilon^{2}\left(p^{-1-s^{*}}+p^{-\bar{\mu}\left(s^{*}, \delta\right)}\right) a_{s^{*}}^{b}+C \varepsilon^{5 / 2}, \\
\left\|\sigma_{\sim}^{\varepsilon} \cdot \underset{\sim}{s}-\underset{\sim}{\sigma^{\varepsilon}}(p) \cdot \underset{\sim}{s}\right\|_{L^{2}\left(P^{\varepsilon}\right)} & \leq C \varepsilon^{5 / 2} p^{-2-s} a_{s}^{1}+C \varepsilon^{3}, \\
\left\|\underset{\sim}{\sigma^{\varepsilon}}-{\underset{\sim}{\sigma}}_{\sim}^{\varepsilon}(p)\right\|_{L^{2}\left(P_{0}^{\varepsilon}\right)} & \leq C \varepsilon^{5 / 2} p^{-2-s} a_{s}^{1}+C \varepsilon^{9 / 2}, \\
\left\|\sigma_{3}^{\varepsilon}-\sigma_{3}^{\varepsilon}(p)\right\|_{L^{2}\left(P^{\varepsilon}\right)} & \leq C \varepsilon^{3 / 2} p^{-1-s} a_{s}+C \varepsilon^{2},
\end{aligned}
$$

where $\underset{\sim}{\sigma^{\varepsilon}}=\underset{\sim}{\nabla} u^{\varepsilon}$

Proof. We prove the fifth estimate only, as the others follow from similar arguments. Using the triangle inequality the following holds:

$\left\|\sigma_{3}^{\varepsilon}-\sigma_{3}^{\varepsilon}(p)\right\|_{L^{2}\left(P^{\varepsilon}\right)} \leq\left\|e_{2}\right\|_{H^{1}\left(P^{\varepsilon}\right)}+\left\|\underline{\Delta}_{2}\right\|_{L^{2}\left(P^{\varepsilon}\right)}+\varepsilon^{3 / 2}\left\|\sigma_{3}^{2}-\sigma_{3}^{2}(p)\right\|_{L^{2}(P)}+\varepsilon\left\|\Upsilon_{3}\right\|_{L^{2}\left(P^{\varepsilon}\right)}$.

From Theorems 2.1 and 2.2, we have that

$$
\left\|e_{2}\right\|_{H^{1}\left(P^{\varepsilon}\right)}+\left\|\underline{\Delta}_{2}\right\|_{L^{2}\left(P^{\varepsilon}\right)} \leq C \varepsilon^{3} .
$$

The estimate

$$
\left\|\Upsilon_{3}\right\|_{L^{2}\left(P^{\varepsilon}\right)} \leq C \varepsilon
$$

comes from Lemmas 2.2 and 3.3. Finally we apply Lemma 3.2 to bound $\| \sigma_{3}^{2}-$ $\sigma_{3}^{2}(p) \|_{L^{2}(P)}$ and the result follows.

Comparing the results of minimum energy models (Theorem 4.3 of Ref. 3) and Theorem 3.1, we see the same rates of convergence in $p$ (with one exception) and in $\varepsilon$ for both classes of models. One should compare the estimates for $\partial_{x_{3}^{\varepsilon}} u^{\varepsilon}(p)$ in the $\mathrm{SP}$ models with the ones for $\sigma_{3}^{\varepsilon}(p)$ in the $\mathrm{SP}^{\prime}$ models, etc. 
Table 1. Rates of convergence of the model error.

\begin{tabular}{ccc}
\hline Quantity & Absolute error & Relative error \\
\hline$u^{\varepsilon}-u^{\varepsilon}(p)$ & $\varepsilon^{5 / 2} p^{-2-s} a_{s}$ & $\nu^{-2} \varepsilon^{2} p^{-2-s} a_{s}$ \\
${\underset{\sim}{\sigma}}_{\sim}^{\varepsilon} \cdot \underset{\sim}{\sim}{\underset{\sim}{\sim}}^{\varepsilon}(p)$ & $\varepsilon^{2} p^{-\bar{\mu}} a_{s}^{b}\left(\varepsilon^{5 / 2} p^{-2-s} a_{s}^{1}\right)$ & $\nu^{-3 / 2} \varepsilon^{3 / 2} p^{-\bar{\mu}} a_{s}^{b}\left(\nu^{-2} \varepsilon^{2} p^{-2-s} a_{s}^{1}\right)$ \\
$\underset{\sim}{\sim} \cdot \underset{\sim}{\sim}-{\underset{\sim}{\sim}}_{\sim}^{\varepsilon}(p) \cdot \underset{\sim}{s}$ & $\varepsilon^{5 / 2} p^{-2-s} a_{s}^{1}$ & $\nu^{-2} \varepsilon^{2} p^{-2-s} a_{s}^{1}$ \\
$\sigma_{3}^{\varepsilon}-\sigma_{3}^{\varepsilon}(p)$ & $\varepsilon^{3 / 2} p^{-1-s} a_{s}$ & $p^{-1-s} a_{s}$ \\
\hline
\end{tabular}

\section{Appendix A. Boundary Layer Results}

Our goal in this Appendix is to prove Lemma 3.3, which estimates the approximation error for the boundary layer part. It is natural then to study the solutions of the following problems defined in the semi-infinite strip $\Sigma$, described below. In this Appendix, we denote an arbitrary point in $\Sigma$ by $\underset{\sim}{\tilde{\rho}}=\left(\tilde{\rho}_{1}, \tilde{\rho}_{2}\right)$.

Let $U \in V(\Sigma)$ and $\underset{\sim}{\Xi} \in \underset{\sim}{S_{0}}(\Sigma)$, where

$$
\begin{aligned}
V(\Sigma)= & \left\{v \in \mathcal{D}^{\prime}(\Sigma):\left(1+\tilde{\rho}_{1}\right)^{-1} v \in L^{2}(\Sigma)\right\}, \\
\underset{\sim}{S}(\Sigma)= & \left\{\underset{\sim}{\tau} \in \mathcal{D}_{\sim}^{\mathcal{D}^{\prime}}(\Sigma):\left(1+\tilde{\rho}_{1}\right) \operatorname{div} \underset{\sim}{\tau} \in L^{2}(\Sigma), \underset{\sim}{\tau} \in{\underset{\sim}{L}}^{2}(\Sigma),\right. \\
& \left.\underset{\sim}{\tau} \cdot \underset{\sim}{n}=0 \text { on } \mathbb{R}^{+} \times\{-1,1\}\right\},
\end{aligned}
$$

be such that

$$
\begin{array}{cc}
\int_{\Sigma} \underset{\sim}{\Xi} \cdot \underset{\sim}{\tau} d \underset{\sim}{\tilde{\rho}}+\int_{\Sigma} U \operatorname{div} \underset{\sim}{\tau} d \underset{\sim}{\tilde{\rho}}=-\int_{\gamma_{0}} U_{0} \tau_{1} d \tilde{\rho}_{2} & \text { for all } \underset{\sim}{\tau} \in \underset{\sim}{S_{0}}(\Sigma), \\
\int_{\Sigma} \operatorname{div} \underset{\sim}{\Xi} v d \underset{\sim}{\tilde{\rho}}=0 & \text { for all } v \in V(\Sigma) .
\end{array}
$$

Here, $\gamma_{0}=\left\{\tilde{\tilde{\rho}} \in \Sigma: \tilde{\rho}_{1}=0\right\}$. The approximate solutions $U(p) \in V(\Sigma, p)$ and $\underset{\sim}{\Xi}(p) \in \underset{\sim}{S_{0}}(\Sigma, p)$ satisfy

$$
\begin{aligned}
& \int_{\Sigma} \underset{\sim}{\Xi}(p) \cdot \underset{\sim}{\tau} d \underset{\sim}{\tilde{\rho}}+\int_{\Sigma} U(p) \operatorname{div} \underset{\sim}{\tau} d \tilde{\sim}=-\int_{\gamma_{0}} U_{0}(p) \tau_{1} d \tilde{\rho}_{2} \quad \text { for all } \underset{\sim}{\tau} \in \underset{\sim}{S_{0}}(\Sigma, p), \\
& \int_{\Sigma} \operatorname{div} \underset{\sim}{\Xi}(p) v d \tilde{\sim}=0 \quad \text { for all } v \in V(\Sigma, p) .
\end{aligned}
$$

To aid the analysis of the difference between the solutions of (A.1) and (A.2), we extend previously defined projection operators to act in the semi-infinite strip $\Sigma$ as well. We shall use an upper-index $\left(\tilde{\rho}_{2}\right)$ to the projection operators notation to indicate that the projection is taking place along each fiber only. So, for instance, if $v \in L^{2}\left(\mathbb{R}^{+} ; \stackrel{\circ}{H}^{1}(-1,1)\right)$, then $\stackrel{\circ}{\pi}_{p}^{1\left(\tilde{\rho}_{2}\right)} v \in L^{2}\left(\mathbb{R}^{+} ; \stackrel{\circ}{\mathbb{P}}_{p}(-1,1)\right)$ is such that

$$
\int_{\Sigma} \partial_{\tilde{\rho}_{2}}\left(\tau-\stackrel{\pi}{\pi}_{p}^{1\left(\tilde{\rho}_{2}\right)} \tau\right) \partial_{\tilde{\rho}_{2}} \tau_{p} d \underset{\sim}{\tilde{\rho}}=0 \quad \text { for all } \tau_{p} \in L^{2}\left(\mathbb{R}^{+} ; \stackrel{\leftrightarrow}{\mathbb{P}}_{p}(-1,1)\right) .
$$

We define $\pi_{p}^{\left(\tilde{\rho}_{2}\right)}$ in a similar fashion. Also, if $\underset{\sim}{\tau}=\left(\tau_{1}, \tau_{2}\right)^{\mathrm{T}}$, then $\underset{\sim}{\prod_{p}} \tau=$ $\left(\pi_{p}^{\left(\tilde{\rho}_{2}\right)} \tau_{1}, \stackrel{\circ}{\pi}_{p+1}^{1\left(\tilde{\rho}_{2}\right)} \tau_{2}\right)^{\mathrm{T}}$. We then have the result below.

Lemma A.1. If $\underset{\sim}{\tau} \in \underset{\sim}{S_{0}}(\Sigma) \cap L^{2}(\Sigma) \times L^{2}\left(\mathbb{R}^{+} ; \stackrel{\circ}{H}^{1}(-1,1)\right)$, then $\pi_{p}^{\left(\tilde{\rho}_{2}\right)} \operatorname{div} \underset{\sim}{\tau}=$ $\operatorname{div} \prod_{\sim} \tau$. 
Proof. It is enough to show that $\int_{\Sigma}\left(\operatorname{div} \underset{\sim}{\prod_{p} \tau}\right) v d \underset{\sim}{\tilde{\rho}}=\int_{\Sigma} \operatorname{div} \underset{\sim}{\tau} v d \tilde{\rho}$ for all $v \in$ $V(\Sigma, p)$. Assuming that $v$ is sufficiently smooth (the general case follows by density), we indeed have

$$
\begin{aligned}
\int_{\Sigma} \operatorname{div} \prod_{\sim} \prod_{\sim} \tau v d \underset{\sim}{\tilde{\rho}} & =\int_{\Sigma}\left(-\pi_{p}^{\left(\tilde{\rho}_{2}\right)} \tau_{1} \partial_{1} v+\partial_{2} \tilde{\pi}_{p+1}^{1\left(\tilde{\rho}_{2}\right)} \tau_{2} v\right) d \underset{\sim}{\tilde{\rho}}+\int_{\gamma_{0}} \pi_{p}^{\left(\tilde{\rho}_{2}\right)} \tau_{1} v d \tilde{\rho}_{2} \\
& =\int_{\Sigma} \partial_{1} \tau_{1} v+\partial_{2} \tau_{2} v d \underset{\sim}{\tilde{\rho}},
\end{aligned}
$$

where we use Lemma 2.3 and integration by parts.

It is important to estimate

$$
\left\|\underset{\sim}{\Xi}-\prod_{\sim} p \underset{\sim}{\Xi}\right\|_{L^{2}(\Sigma)}^{2}=\left\|\Xi_{1}-\pi_{p}^{\left(\tilde{\rho}_{2}\right)} \Xi_{1}\right\|_{L^{2}(\Sigma)}^{2}+\left\|\Xi_{2}-\stackrel{\circ}{\pi}_{p+1}^{1\left(\tilde{\rho}_{2}\right)} \Xi_{2}\right\|_{L^{2}(\Sigma)}^{2} .
$$

The error due to the mixed approximation depends on the regularity of the solution. The convergence rate defined below reflects that.

Definition A.1. For $U_{0} \in H^{r_{0}}(-1,1)$ with $r_{0}>3 / 2$, and $N$ as in $(3.5)$, if there exists a minimum integer $m \in\left\{1, \ldots, N\left(r_{0}+1 / 2\right)\right\}$ such that $\left|\partial_{2}^{2 m-1} U_{0}(-1)\right|+$ $\left|\partial_{2}^{2 m-1} U_{0}(1)\right| \neq 0$, let $\bar{\gamma}\left(r_{0}, \delta\right)=\min \left\{4 m-3-\delta, r_{0}-1 / 2\right\}$, otherwise let $\bar{\gamma}\left(r_{0}, \delta\right)=$ $r_{0}-1 / 2$.

The solution $U$ for (A.1) has a singular behavior at the corners of the semiinfinite strip. To study the approximation rates for $U$, it is useful to decompose this solution in its singular and "smooth" parts. We describe the singular behavior of the solution of (A.1), by introducing in $\Sigma$ two polar coordinate systems, $\left(r_{l}, \theta_{l}\right)$, $l=1,2$ relative to the vertices $P_{1}=(0,1)$ and $P_{2}=(0,-1)$. The convention is that $r_{l}$ gives the distance to $P_{l}$ and the angle $\theta_{l} \in[0, \pi / 2]$ increases counterclockwise, so points lying on $\gamma_{0}$ have $\theta_{1}=0$ and $\theta_{2}=\pi / 2$.

The next theorem, ${ }^{17}$ shows a decomposition of the solution $U$ in singular and smooth parts and it will be of great use henceforward.

Theorem A.1. Let $U \in V(\Sigma)$ be the solution of (A.1) with $r_{0}>3 / 2$ such that $r_{0}+1 / 2$ is not an even integer. Then there exist constants $c_{j}$ such that

$$
U=U_{S}+W, \quad U_{S}=\check{\chi} \sum_{l=1}^{2} \sum_{j=1}^{N\left(r_{0}+1 / 2\right)} c_{j} \partial_{2}^{(2 j-1)} U_{0}\left((-1)^{l+1}\right) v_{l}^{j},
$$

where $\check{\chi}$ is a smooth cutoff function that equals the identity for $x_{1}<1$ and vanishes for $x_{1}>2, N$ is as in (3.5), and

$$
\begin{aligned}
& v_{1}^{j}=\left[\theta_{1} \cos \left((2 j-1) \theta_{1}\right)+\log r_{1} \sin \left((2 j-1) \theta_{1}\right)\right] r_{1}^{(2 j-1)}, \\
& v_{2}^{j}=\left[\left(\frac{\pi}{2}-\theta_{2}\right) \sin \left((2 j-1) \theta_{2}\right)+\log r_{2} \cos \left((2 j-1) \theta_{2}\right)\right] r_{2}^{(2 j-1)} .
\end{aligned}
$$

Furthermore, $\|W\|_{H^{r_{0}+1 / 2}(\Sigma)} \leq c\left\|U_{0}\right\|_{H^{r_{0}}\left(\gamma_{0}\right)}$ for some constant $c$. 
With the above decomposition result, it is possible to prove ${ }^{3}$ that

$$
\left\|\Xi_{1}-\pi_{p}^{\left(\tilde{\rho}_{2}\right)} \Xi_{1}\right\|_{L^{2}(\Sigma)} \leq C p^{-\bar{\gamma}\left(r_{0}, \delta\right)}\left\|U_{0}\right\|_{H^{r_{0}(-1,1)}} .
$$

Unfortunately, the estimates for $\Xi_{2}$ do not come so easily, since $\stackrel{\circ}{\pi}_{p+1}^{1\left(\tilde{\rho}_{2}\right)}$ does not necessarily yield the best approximation in the $L^{2}$ norm. We divide the error analysis in two cases. We first analyze the general situation and then improve the result for particular conditions.

Lemma A.2. For any arbitrarily small positive real number $\delta$, there exists a constant $c$ such that

$$
\left\|\Xi_{2}-\stackrel{\circ}{\pi}_{p+1}^{1\left(\tilde{\rho}_{2}\right)} \Xi_{2}\right\|_{L^{2}(\Sigma)} \leq c p^{-1+\delta}\left\|U_{0}\right\|_{H^{3 / 2}(-1,1)}
$$

Proof. We show only the main inequalities involved. In general, $U \in H^{2-\delta}(\Sigma)$. Hence

$$
\left\|\partial_{2} U-\stackrel{\circ}{\pi}_{p+1}^{1\left(\tilde{\rho}_{2}\right)} \partial_{2} U\right\|_{L^{2}(\Sigma)} \leq c p^{-1+\delta}\|U\|_{L^{2}\left(\mathbb{R}^{+} ; H^{2-\delta}(-1,1)\right)} \leq c p^{-1+\delta}\left\|U_{0}\right\|_{H^{3 / 2}(-1,1)} .
$$

See Ref. 19 for further details.

Assume now that

$$
\left|\partial_{2} U_{0}(-1)\right|+\left|\partial_{2} U_{0}(1)\right|=0
$$

If $r_{0} \in(3 / 2,5 / 2)$, then

$$
\left\|\Xi_{2} U-\stackrel{\circ}{\pi}_{p+1}^{1\left(\tilde{\rho}_{2}\right)} \Xi_{2}\right\|_{L^{2}(\Sigma)} \leq c p^{-r_{0}-1 / 2}\left\|U_{0}\right\|_{H^{r_{0}(-1,1)}} .
$$

Otherwise, using a duality argument, ${ }^{7}$

$$
\begin{aligned}
\left\|\Xi_{2}-\stackrel{\circ}{\pi}_{p+1}^{1\left(\tilde{\rho}_{2}\right)} \Xi_{2}\right\|_{L^{2}(\Sigma)} & \leq c p^{-1}\left\|\partial_{2} \Xi_{2}-\partial_{2} \stackrel{\circ}{\pi}_{p+1}^{1\left(\tilde{\rho}_{2}\right)} \Xi_{2}\right\|_{L^{2}(\Sigma)} \\
& =c p^{-1}\left\|\partial_{2} \Xi_{2}-\pi_{p}^{\left(\tilde{\rho}_{2}\right)} \partial_{2} \Xi_{2}\right\|_{L^{2}(\Sigma)},
\end{aligned}
$$

by Lemma 2.3. Note that $\Xi_{2}$ solve the following Dirichlet problem:

$$
\begin{array}{cl}
\Delta \Xi_{2}=0 & \text { in } \Sigma, \\
\Xi_{2}=0 \quad \text { on } \mathbb{R} \times\{-1,1\}, & \Xi_{2}=\partial_{2} U_{0} \quad \text { on } \gamma_{0},
\end{array}
$$

where $U_{0} \in H^{r_{0}}(-1,1)$.

A way to obtain a good approximation for $\Xi_{2}$ is, as before, by splitting $\Xi_{2}$ in singular and smooth parts, and seeking approximations for both. In the next two results we do exactly that. The following theorem comes from Ref. 17 .

Theorem A.2. Let $\Xi_{2}$ be the solution of (A.10) with $r_{0}>5 / 2$ such that $r_{0}+1 / 2$ is not an even integer, and assume that (A.7) holds. Then there exist constants $c_{j}, c$ such that

$$
\Xi_{2}=\Xi_{2_{S}}+W_{2}, \quad \Xi_{2_{S}}=\check{\chi} \sum_{l=1}^{2} \sum_{j=2}^{N\left(r_{0}+1 / 2\right)} c_{j} \partial_{2}^{2 j-1} U_{0}\left((-1)^{l+1}\right) \bar{v}_{l}^{j-1},
$$


where

$$
\begin{aligned}
& \bar{v}_{1}^{j}=\left[\left(\frac{\pi}{2}-\theta_{1}\right) \cos \left(2 j \theta_{1}\right)+\log r_{1} \sin \left(2 j \theta_{1}\right)\right] r_{1}^{2 j}, \\
& \bar{v}_{2}^{j}=\left[\theta_{2} \cos \left(2 j \theta_{2}\right)+\log r_{2} \sin \left(2 j \theta_{2}\right)\right] r_{2}^{2 j},
\end{aligned}
$$

and $\left\|W_{2}\right\|_{H^{r_{0}-1 / 2(\Sigma)}} \leq c\left\|U_{0}\right\|_{H^{r_{0}\left(\gamma_{0}\right)}}$.

The next lemma shows the approximation rates to the above defined singular functions. It follows from an application of the ideas of Dorr, ${ }^{13,14}$ and the Remark 6.3 of Bernardi and Maday. ${ }^{7}$ See Ref. 19 for a description of how such convergence rates can be obtained.

Lemma A.3. Let $v(r, \theta)=\check{\chi} r^{\alpha}\left[\xi_{1}(\theta)+\xi_{2}(\theta) \log r\right]$, where $\xi_{1}, \xi_{2} \in C^{\infty}([0, \pi / 2])$, and $\alpha$ is a non-negative real number. Then, for every $\delta$, there exists a constant $c$ such that

$$
\left\|v-\pi_{p}^{\left(\tilde{\rho}_{2}\right)} v\right\|_{H^{1}(\Sigma)} \leq c p^{-2 \alpha+\delta} .
$$

With the above lemma, it is easy to estimate approximation errors to $\Xi_{2}$, as we show below.

Lemma A.4. Assume that the hypotheses of Theorem A.2 hold. Then

$$
\left|\partial_{2} W_{2}-\partial_{2} \stackrel{\circ}{\pi}_{p}^{1\left(\tilde{\rho}_{2}\right)} W_{2}\right|_{L^{2}(\Sigma)} \leq c p^{3 / 2-r_{0}}\left\|U_{0}\right\|_{H^{r_{0}}\left(\gamma_{0}\right)} .
$$

Also, if $\Xi_{2_{S}}$ is not the zero function, then for each arbitrarily small $\delta>0$ there exists a constant $c$ such that

$$
\left\|\Xi_{2_{S}}-\pi_{p}^{\left(\tilde{\rho}_{2}\right)} \Xi_{2_{S}}\right\|_{H^{1}(\Sigma)} \leq c p^{-4 m+4+\delta}\left\|U_{0}\right\|_{H^{r_{0}}\left(\gamma_{0}\right)},
$$

where $m \in\left\{2, \ldots, N\left(r_{0}+\frac{1}{2}\right)\right\}$ is the minimum integer such that

$$
\left|\partial_{2}^{(2 m-1)} U_{0}(-1)\right|+\left|\partial_{2}^{(2 m-1)} U_{0}(1)\right| \neq 0 .
$$

Proof. Inequality (A.12) follows from spectral approximation properties, i.e.

$$
\left|\partial_{2} W_{2}-\partial_{2} \stackrel{\circ}{\pi}_{p}^{1\left(\tilde{\rho}_{2}\right)} W_{2}\right|_{L^{2}(\Sigma)} \leq c p^{3 / 2-r_{0}}\left\|W_{2}\right\|_{L^{2}\left(\mathbb{R}^{+} ; H^{r_{0}-1 / 2}\right)} \leq c p^{3 / 2-r_{0}}\left\|U_{0}\right\|_{H^{r_{0}}\left(\gamma_{0}\right)},
$$

where the last inequality follows from Theorem A.2. Estimate (A.13) follows from the definition of $\Xi_{2_{S}}$, and Lemma A.3.

Now we are ready to estimate $\left\|\underset{\sim}{\Xi}-\prod_{p} \underset{\sim}{\Xi}\right\|_{L^{2}(\Sigma)}$.

Lemma A.5. For any $r_{0}>3 / 2$ such that $r_{0}+1 / 2$ is not an even integer, and any arbitrarily small $\delta>0$, there exists a constant $c$ such that

$$
\begin{aligned}
\left\|\Xi_{1}-\stackrel{\circ}{\pi}_{p+1}^{1\left(\tilde{\rho}_{2}\right)} \Xi_{1}\right\|_{L^{2}(\Sigma)} & \leq c p^{-\bar{\gamma}\left(r_{0}, \delta\right)}\left\|U_{0}\right\|_{H^{r_{0}}(-1,1)}, \\
\left\|\Xi_{2}-\stackrel{\pi}{\pi}_{p+1}^{1\left(\tilde{\rho}_{2}\right)} \Xi_{2}\right\|_{L^{2}(\Sigma)} & \leq c p^{-\bar{\gamma}\left(r_{0}, \delta\right)}\left\|U_{0}\right\|_{H^{r_{0}}(-1,1)}, \\
\left\|\underset{\sim}{\Xi}-\underset{\sim}{\prod_{p}} \underset{\sim}{\Xi}\right\|_{L^{2}(\Sigma)} & \leq c p^{-\bar{\gamma}\left(r_{0}, \delta\right)}\left\|U_{0}\right\|_{H^{r_{0}}(-1,1)},
\end{aligned}
$$

where $\bar{\gamma}$ is as in Definition A.1. 
Proof. The first bound follows immediately from (A.5). The second estimate follows, for $r_{0} \in(3 / 2,5 / 2]$, from (A.6) and (A.8). For $r_{0}>5 / 2$, note only that, from (A.9) and Theorem A.2,

$$
\begin{aligned}
& \left\|\Xi_{2}-\stackrel{\circ}{\pi}_{p+1}^{1\left(\tilde{\rho}_{2}\right)} \Xi_{2}\right\|_{L^{2}(\Sigma)} \\
& \quad \leq c p^{-1}\left(\left\|\partial_{2} \Xi_{2 S}-\partial_{2} \stackrel{\circ}{\pi}_{p+1}^{1\left(\tilde{\rho}_{2}\right)} \Xi_{2 S}\right\|_{L^{2}(\Sigma)}+\left\|\partial_{2} W_{2}-\partial_{2} \stackrel{\circ}{\pi}_{p+1}^{1\left(\tilde{\rho}_{2}\right)} W_{2}\right\|_{L^{2}(\Sigma)}\right) .
\end{aligned}
$$

Next, use (A.12), the inequality

$$
\left\|\partial_{2} \Xi_{2 S}-\partial_{2} \stackrel{\circ}{p+1}_{\left.p+\tilde{\rho}_{2}\right)}^{(\tilde{I}} \Xi_{2 S}\right\|_{L^{2}(\Sigma)} \leq\left\|\Xi_{2_{S}}-\pi_{p}^{\left(\tilde{\rho}_{2}\right)} \Xi_{2_{S}}\right\|_{H^{1}(\Sigma)},
$$

and (A.13). Finally, the third estimate of this lemma follows from (A.3).

The next theorem estimates the mixed approximation.

Theorem A.3. Assume that $U \in V(\Sigma), \Xi \in S_{0}(\Sigma)$ solve (A.1) and $U(p) \in V(\Sigma, p)$, $\Xi(p) \in S_{0}(\Sigma, p)$ solve (A.2). Then for any non-negative real number $r_{0}>3 / 2$ such that $r_{0}+1 / 2$ is not an even integer, and any arbitrarily small $\delta>0$, there exists a constant $c$ such that

$$
\|\underset{\sim}{\Xi}-\underset{\sim}{\Xi}(p)\|_{\sim}^{L^{2}(\Sigma)} \leq c\left(\left\|U_{0}-U_{0}(p)\right\|_{H^{1 / 2}\left(\gamma_{0}\right)}+p^{-\bar{\gamma}\left(r_{0}, \delta\right)}\left\|U_{0}(p)\right\|_{H^{r_{0}}\left(\gamma_{0}\right)}\right) .
$$

Proof. Let $\tilde{U} \in V(\Sigma)$ and $\underset{\sim}{\tilde{\Xi}} \in \underset{\sim}{S_{0}}(\Sigma)$ be such that

$$
\begin{aligned}
\int_{\Sigma} \underset{\sim}{\tilde{\Xi}} \cdot \underset{\sim}{\tau} d \tilde{\rho}+\int_{\Sigma} \tilde{U} \operatorname{div} \underset{\sim}{\tau} d \underset{\sim}{\tilde{\rho}}=\int_{\gamma_{0}} U_{0}(p) \tau_{1} d \tilde{\rho}_{2} & \text { for all } \underset{\sim}{\tau} \in \underset{\sim}{S_{0}(\Sigma),} \\
\int_{\Sigma} \operatorname{div} \underset{\sim}{\tilde{\Xi}} v d \tilde{\rho}=0 & \text { for all } v \in V(\Sigma) .
\end{aligned}
$$

Then,

$$
\|\underset{\sim}{\Xi}-\underset{\sim}{\tilde{\Xi}}\|_{L^{2}(\Sigma)} \leq c\left\|U_{0}-U_{0}(p)\right\|_{H^{1 / 2}\left(\gamma_{0}\right)} .
$$

To conclude the estimate, we use Lemma A.1 as follows:

$$
\begin{aligned}
& \int_{\Sigma}[\underset{\sim}{\tilde{\Xi}}-\underset{\sim}{\Xi}(p)] \prod_{\sim} p \underset{\sim}{\underset{\Xi}{\Xi}} d \tilde{\sim}=-\int_{\Sigma}[\tilde{U}-U(p)] \operatorname{div} \prod_{\sim} p \underset{\sim}{\tilde{\Xi}} d \tilde{\rho} \\
& =-\int_{\Sigma}[\tilde{U}-U(p)] \pi_{p}^{\left(\tilde{\rho}_{2}\right)} \operatorname{div} \underset{\sim}{\tilde{\Xi}} d \tilde{\sim}=-\int_{\Sigma}[\tilde{U}-U(p)] \operatorname{div} \underset{\sim}{\Xi}(p) d \underset{\sim}{\tilde{\rho}} \\
& =\int_{\Sigma}[\underset{\sim}{\tilde{\Xi}}-\underset{\sim}{\Xi}(p)] \underset{\sim}{\Xi}(p) d \tilde{\rho},
\end{aligned}
$$

since $\pi_{p}^{\left(\tilde{\rho}_{2}\right)} \operatorname{div} \underset{\sim}{\tilde{\Xi}}=\operatorname{div} \underset{\sim}{\Xi}(p)$, and then

$$
\|\underset{\sim}{\tilde{\Xi}}-\underset{\sim}{\Xi}(p)\|_{L^{2}(\Sigma)}^{2}=\int_{\Sigma}[\underset{\sim}{\tilde{\Xi}}-\underset{\sim}{\Xi}(p)]\left[\underset{\sim}{\tilde{\Xi}}-\prod_{\sim} p \underset{\sim}{\tilde{\Xi}}\right] d \tilde{\sim} \leq\|\underset{\sim}{\tilde{\Xi}}-\underset{\sim}{\Xi}(p)\|_{L^{2}(\Sigma)}\left\|\underset{\sim}{\tilde{\Xi}}-\prod_{\sim} p \underset{\sim}{\tilde{\Xi}}\right\|_{L^{2}(\Sigma)} .
$$


Next, since $\prod_{\sim}$ is a bounded operator,

$$
\begin{aligned}
& \|\underset{\sim}{\tilde{\Xi}}-\underset{\sim}{\Xi}(p)\|_{L^{2}(\Sigma)} \leq\left\|\underset{\sim}{\tilde{\Xi}}-\prod_{\sim} p \underset{\sim}{\tilde{\Xi}}\right\|_{L^{2}(\Sigma)} \\
& \leq\|\underset{\sim}{\tilde{\Xi}}-\underset{\sim}{\Xi}\|_{L^{2}(\Sigma)}+\left\|\underset{\sim}{\Xi}-\underset{\sim}{\prod_{p}} \underset{\sim}{\Xi}\right\|_{L^{2}(\Sigma)}+\left\|\prod_{\sim} p \underset{\sim}{\Xi}-\prod_{\sim} p \underset{\sim}{\underset{\Xi}{\Xi}}\right\|_{L^{2}(\Sigma)} \\
& \leq c\|\underset{\sim}{\tilde{\Xi}}-\underset{\sim}{\Xi}\|_{L^{2}(\Sigma)}+\left\|\underset{\sim}{\Xi}-\prod_{\sim} p \underset{\sim}{\Xi}\right\|_{L^{2}(\Sigma)} \\
& \leq c\left\|U_{0}-U_{0}(p)\right\|_{H^{1 / 2}\left(\gamma_{0}\right)}+\left\|\underset{\sim}{\Xi}-\prod_{\sim} \underset{\sim}{\Xi}\right\|_{L^{2}(\Sigma)} \\
& \leq c\left\|U_{0}-U_{0}(p)\right\|_{H^{1 / 2}\left(\gamma_{0}\right)}+c p^{-\bar{\gamma}\left(r_{0}, \delta\right)}\left\|U_{0}(p)\right\|_{H^{r_{0}}\left(\gamma_{0}\right)},
\end{aligned}
$$

where we used Lemma A.5 to obtain the last inequality. The theorem follows from (A.14) and the inequality above.

Comparing Theorem A.3 with Theorem A.5 of Ref. 3 we see that the estimates for the mixed approximations are worse than the estimates for the Galerkin approximation. For instance, if $U_{0}\left(\tilde{\rho}_{2}\right)=U_{0}(p)\left(\tilde{\rho}_{2}\right)=\tilde{\rho}_{2}$, then we can bound the error coming from the mixed methods as

$$
\|\underset{\sim}{\Xi}-\underset{\sim}{\Xi}(p)\|_{L^{2}(\Sigma)} \leq c p^{-1+\delta}\left\|U_{0}\right\|_{H^{r_{0}}\left(\gamma_{0}\right)},
$$

while we bound the error from the Galerkin methods as

$$
\|\underset{\sim}{\nabla} U-\underset{\sim}{\nabla} U(p)\|_{L^{2}(\Sigma)} \leq c p^{-2+\delta}\left\|U_{0}\right\|_{H^{r_{0}}\left(\gamma_{0}\right)} .
$$

It is not clear whether the upper bound of Theorem A.3 is sharp or not, and, to the best of our knowledge, there is no numerical evidence to support either case. The culprit for this possible loss of accuracy is the use of a duality argument. In fact, Eriksson ${ }^{15}$ worked out a one-dimensional example and showed that the duality argument does not yield the best possible error estimate for the $p$-method.

Proof. (of Lemma 3.3) From Definitions 3.2, Appendix A.1 and Theorem A.3, we have that, for each $\underset{\sim}{x} \in \partial \Omega$,

$$
\begin{aligned}
& \left\|\tilde{\Xi}_{n}^{2}-\tilde{\Xi}_{n}^{2}(p)\right\|_{L^{2}(\Sigma)}+\left\|\tilde{\Xi}_{3}^{2}-\tilde{\Xi}_{3}^{2}(p)\right\|_{L^{2}(\Sigma)} \\
& \left.\quad \leq c\left(\| u^{2}(\underset{\sim}{x}, \cdot)-u^{2}(p) \underset{\sim}{x}, \cdot\right)\left\|_{H^{1 / 2}(-1,1)}+p^{-\bar{\mu}(s, \delta)}\right\| u^{2}(\underset{\sim}{x}, \cdot) \|_{H^{s+2}(-1,1)}\right) .
\end{aligned}
$$

After changing coordinates, and using (A.16) we see that

$$
\begin{aligned}
\left\|\Upsilon_{n}\right\|_{L^{2}\left(P^{\varepsilon}\right)}+\left\|\Upsilon_{3}\right\|_{L^{2}\left(P^{\varepsilon}\right)} \leq & c \varepsilon\left(\left\|u^{2}-u^{2}(p)\right\|_{L^{2}\left(\partial \Omega ; H^{1 / 2}(-1,1)\right)}\right. \\
& \left.+p^{-\bar{\mu}(s, \delta)}\left\|u^{2}\right\|_{L^{2}\left(\partial \Omega ; H^{s+2}(-1,1)\right)}\right) .
\end{aligned}
$$

From Lemma 3.1 and using (3.3), we gather that

$$
\left\|u^{2}-u^{2}(p)\right\|_{H^{1 / 2}\left(\partial P_{L}\right)} \leq c p^{-1-s} a_{b}^{s}
$$

and the result follows. 


\section{Acknowledgments}

The author would like to thank Douglas N. Arnold for several helpful suggestions he gave during the completion of this work. Support from CNPq-Brazil is gratefully acknowledge.

\section{References}

1. S. M. Alessandrini, D. N. Arnold, R. S. Falk and A. L. Madureira, Derivation and justification of plate models by variational methods, in Plates and Shells (Amer. Math. Soc., 1999), pp. 1-20.

2. D. N. Arnold and R. S. Falk, Asymptotic analysis of the boundary layer for the Reissner-Mindlin plate model, SIAM J. Math. Anal. 27 (1996) 468-514.

3. D. N. Arnold and A. L. Madureira, Asymptotic estimates of hierarchical modeling, Math. Mod. Meth. Appl. Sci. 13 (2003) 1325-1350.

4. I. Babuška and M. Suri, The optimal convergence rate of the $p$-version of the finite element method, SIAM J. Numer. Anal. 24 (1987) 750-776.

5. I. Babuška and L. Li, Hierarchical modelling of plates, Comput. Structures 40 (1991) 419-430.

6. I. Babuška and L. Li, The problem of plate modeling: Theoretical and computational results, Comput. Meth. Appl. Mech. Engrg. 100 (1992) 249-273.

7. C. Bernardi and Y. Maday, Spectral Methods, Handbook of Numerical Analysis, Vol. V eds. P. G. Ciarlet and J. L. Lions (Elsevier, 1997).

8. F. Brezzi and M. Fortin, Mixed and Hybrid Finite Element Methods, Springer Series in Computational Mathematics, Vol. 15 (Springer-Verlag, 1991).

9. C. Chen, Asymptotic convergence rates for the Kirchhoff plate model, Ph.D. Dissertation, The Pennsylvania State University (1995).

10. P. G. Ciarlet, Mathematical Elasticity, Vol. II: Theory of Plates, Studies in Mathematics and Its Applications, Vol. 27 (Elsevier, 1997).

11. M. Dauge and I. Gruais, Asymptotics of arbitrary order for a thin elastic clamped plate, I: Optimal error estimates, Asympt. Anal. 13 (1996) 167-197.

12. M. Dauge, I. Gruais and A. Rössle, The influence of lateral boundary conditions on the asymptotics in thin elastic plates, SIAM J. Math. Anal. 31 (2000) 305-345.

13. M. Dorr, The approximation theory for the $p$-version of the finite element method, SIAM J. Numer. Anal. 21 (1984) 1180-1207.

14. M. Dorr, The approximation of solutions of elliptic boundary-value problems via the $p$-version of the finite element method, SIAM J. Numer. Anal. 23 (1986) 58-77.

15. K. Eriksson, Some error estimates for the $p$-version of the finite element method, SIAM J. Numer. Anal. 23 (1986) 403-411.

16. C. O. Horgan and J. K. Knowles, Recent developments concerning Saint-Venant's principle, Adv. Appl. Mech. 23 (1983) 179-269.

17. R. B. Kellogg, Notes on piecewise smooth elliptic boundary value problems, Institute for Physical Science and Technology, Technical Note BN-1137 (1992).

18. K. H. Lo, R. M. Christensen and E. M. Wu, A high-order theory of plate deformation, J. Appl. Mech. 46 (1977) 663-676.

19. A. L. Madureira, Asymptotics and hierarchical modeling of thin plates, Ph.D. Dissertation, The Pennsylvania State University (1999).

20. W. G. Mazja, S. A. Nazarow and B. A. Plamenewski, Asymptotische Theorie Ellipscher Randwertaufgaben in Singulär gestörten Gebieten I (Akademie Verlag, 1991). 
21. B. Miara, Optimal spectral approximation in linearized plate theory, Appl. Anal. 31 (1989) 291-307.

22. D. S. Mitrinović, J. E. Pečarić and A. M. Fink, Inequalities Involving Functions and their Integrals and Derivatives (Kluwer, 1991).

23. D. Morgenstern, Herleitung der plattentheorie aus der dreidimensionalen elastizitatstheorie, Arch. Rational Mech. Anal. 4 (1959) 145-152.

24. O. Ovaskainen and J. Pitkäranta, An energy method approach to the problem of elastic strip, SIAM J. Appl. Math. 58 (1998) 999-1021.

25. A. Rössle, M. B. W. Wendland and E. Ramm, On the mathematical foundation of the (1,1,2)-plate model, Universität Stuttgart, Sonderforschungsbereich 404 (1997).

26. C. Schwab, Wavelets, multilevel methods and elliptic PDEs, in Hierarchic Modelling in Mechanics, eds. M. Ainsworth, J. Levesley, W. A. Light and M. Marletta (Oxford Univ. Press, 1997), pp. 85-160.

27. M. Vogelius and I. Babuška, On a dimensional reduction method I. The optimal selection of basis functions, Math. Comput. 37 (1981) 31-46.

28. M. Vogelius and I. Babuška, On a dimensional reduction method II. Some approximation-theoretic results, Math. Comput. 37 (1981) 47-68.

29. M. Vogelius and I. Babuška, On a dimensional reduction method III. A posteriori error estimation and an adaptive approach, Math. Comput. 37 (1981) 361-384. 